International Journal of Fracture manuscript No.

(will be inserted by the editor)

\title{
Analysis of fracture nucleation around wellbores
}

\author{
L. Jeannin ${ }^{1} \cdot$ L. Dormieux ${ }^{2} \cdot$ E. Lemarchand ${ }^{2 *} \cdot$ T. Carlioz $^{2}$
}

Received: date / Accepted: date

\begin{abstract}
Crack nucleation has been the subject of important contributions in the two last decades. Starting from the double criterion of [14], the present paper suggests that an energy criterion is sufficient for the prediction of the loading level that triggers nucleation as well as its geometrical characteristics. In view of application to saturated porous materials, the role of the pore fluid during nucleation is considered. In case of low fluid compressibility, the presence of the fluid delays the brittle failure usually associated with nucleation, as long as the fluid pressure remains above the saturation vapour pressure. The energy nucleation criterion is then applied to discuss the formation of breakouts around wellbores subjected to remote compressive stresses.
\end{abstract}

Keywords crack nucleation, saturated porous media, stability analysis, damage model, wellbore

\section{Introduction}

The concentration of stress around wellbores during drilling can lead to both compressive failures known as stress-induced breakouts and to tensile cracks. Wellbore imaging devices make it possible to obtain direct information on the distribution and orientation of fractures and cracks encountered at dpth in wells. The observation of the failure patterns provides information on the stress regime around the well, and more particularly on the orientation and magnitude of the principal stresses.

A full understanding of compressive and tensile failure is therefore important for evaluation of stress field principal directions and wellbore stability issue [30]. Breakouts are generally interpreted in the framework of elasto-plasticity with a Mohr-Coulomb yield surface, while tensile cracks induced by mud overpessure during drilling are interpreted as a brittle failure. We take in this study a different point of view: the drilled rock is regarded as an elastic brittle material in which the failure mechanism is based on the nucleation of cracks. In particular, this has the advantage that no assumption on stresses acting on failure lines will be required.

Let us model the wellbore in the framework of plane strains as a circular hole in an infinite plane with given remote stresses at infinity. For example, let a compressive $\sigma_{y y}^{\infty}<0$ be applied at infinity. Under this loading, it is well known that tensile stresses appear in the vicinity of the hole along the $O y$-axis in the direction of $\boldsymbol{e}_{x}$. The propagation of symmetric tensile (mode I) cracks (see figure 1, left) has been extensively studied both experimentally and theoretically in the literature (see e.g. [28]). Apart from this first brittle failure mechanism, breakouts in the perpendicular direction (see figure 1, middle) constitute a possible alternative failure mechanism, at least theoretically. It would imply the nucleation of two cracks in mode II.

1 Storengy, Immeuble Djiin, 12 rue Raoul Nordling, 92274, Bois-Colombes Cedex, France

2 Laboratoire Navier, Ecole des Ponts Paristech, 6 et 8, avenue Blaise Pascal 77455 Marne-la-Vallée, France

$(*)$ Corresponding author 
One could also imagine the formation of two symmetric breakouts in a combination of modes I and II (see figure 1, right).
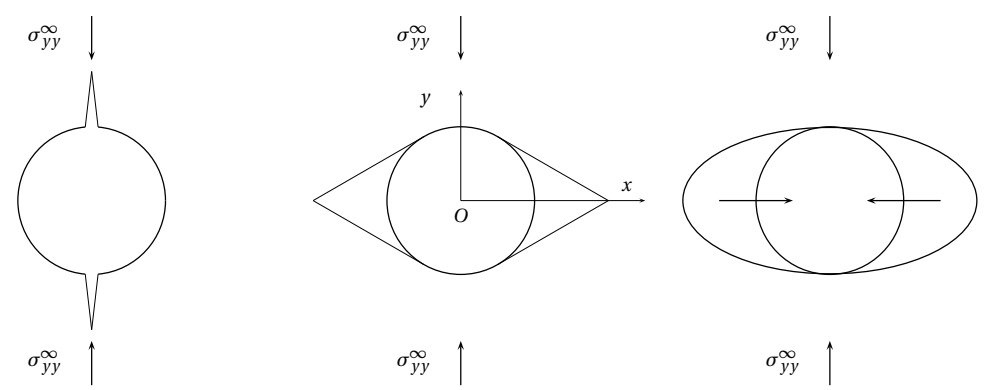

Fig. 1 failure mechanisms under uniaxial compression: mode I (left), mode II (middle), mixed modes (right)

The purpose of a nucleation theory is to predict the occurrence of a crack of finite size in a quasiinstantaneous way in an elastic material. The aim of the present paper is to provide a theoretical framework that may likely explain both tensile cracks and breakouts in the engineering context of wellbores. From a quantitative point of view, such a theory should be able to predict the loading level that induces nucleation as well as the extent of the cracks that are nucleated. In particular, it should be able to discuss which of the failure mechanisms presented at figure 1 is likely to take place.

By definition, the nucleation is the transition from the sound material to the cracked material, when a critical loading level is reached. By nature, it can be viewed as the propagation of a crack, starting from a length equal to 0 , but in a very short period of time. In the variational approach (e.g. [5], [6]), the crack is viewed as the final state of some region of the structure in which the material has reached a full damage level. The determination of the loading required for the crack nucleation and of the geometry of the nucleated crack is achieved by a double variational principle concerning the displacement on the one hand and the damage on the other hand. The energy function to which this variational procedure is applied comprises a term accounting for damage gradient in the medium. This approach requires by nature to follow the evolution of the system in a step-by-step procedure. In contrast, the so-called double criterion first proposed in [14] does not go through the time process leading to nucleation. The emphasis is put on the fact that the propagation that produces the crack is not stable in the sense that the crack length increases under constant loading as long as it has not reached the nucleation length, if the latter exists. Therefore, the idea is to deal with nucleation as a discontinuity with respect to time in the state of the system. It relies upon an energy balance between the initiation of the nucleation and its end which includes a variation of the potential energy $\delta E_{p}$, of the kinetic energy $\delta K$ and a dissipation $G_{c} \delta S$ proportional to the created crack area $\delta S$ ( $G_{c}$ is the fracture energy). Observing that the increment of the kinetic energy is necessarily a positive number if the initial state is at equilibrium, a very simple necessary condition of nucleation is derived in the form of an energy criterion which states that the release of potential energy should be greater than the energy dissipated in the crack nucleation:

$$
-\delta E_{p} \geq G_{c} \delta S
$$

In [14], it is suggested that (1) must be combined with a stress criterion to be fulfilled in the area where the crack is nucleated in order to determine the critical loading level of nucleation as well as its extent. This approach is therefore often referred to as the double criterion method. More recently, [15] however argued that the energy criterion was sufficient for characterizing the occurence and the extent of nucleation. This new methodology was applied to study fracture nucleation, that occurs during tunnelling in the context of nuclear waste storage.

First, the mechanical and thermodynamical framework of the energy criterion for nucleation is presented as well as the general ideas for the practical implementation. The specific question of nucleation in liquid saturated porous media is then addressed with special emphasis on mode I crack nucleation. We then apply this general theoretical framework to the nucleation of tensile or compressive cracks around a hole in compression. 


\section{Criterion for nucleation}

Let $\Omega$ denote the mechanical system in which possible crack nucleation is considered. The geometry of the crack is described by a formal parameter $\ell$. This is for instance the length in $2 \mathrm{D}$ or the area in $3 \mathrm{D}$. It controls the energy $G_{c} \ell$ that is dissipated during nucleation. During the nucleation process, $\ell$ increases from 0 to a limit value $\ell^{*}$ for which stability is recovered under the loading that has triggered the nucleation.

The created crack $\mathscr{C}(\ell)$ is viewed as a material system since it is the residual state of a subdomain of $\Omega$ in which the material undergoes a full damage process at the end of which it has lost its stiffness. As already mentionned, this description is also underlying the variational approach (e.g. [5]). As compared to its length, $\mathscr{C}(\ell)$ possesses some non zero thickness, though the latter is infinitesimal. It is therefore relevant to define its thermodynamical and mechanical variables and functions, such as strain, stress, stiffness tensors and internal energy. From a mathematical point of view, the 2-dimensional description of $\mathscr{C}(\ell)$ by a surface in 3D ( respectively 1-dimensional in 2D) is of course the most convenient. It should however be kept in mind that this is only a mathematical idealization. For instance, nucleation viewed as a full damage process removes the elastic stiffness of the solid inside $\mathscr{C}(\ell)$ but not the heat capacity, so that the material keeps its ability to store internal energy. Besides, the fluid phase in $\mathscr{C}(\ell)$ has a finite compressibility. In undrained conditions, it therefore contributes to the spherical part of the undrained stiffness tensor, and this contribution is not subjected to damage.

Let $\Omega(\ell)=\Omega \backslash \mathscr{C}(\ell)$ denote the complementary of the crack in $\Omega$. By definition, as long as $\dot{\ell}=0, \Omega(\ell)$ undergoes reversible evolutions only.

Crack nucleation is regarded as a sudden phenomenon as compared to the characteristic times of fluid and heat diffusion (see e.g. [17]). Fluid and heat flow are therefore neglected at the time scale of nucleation. Apart from this point, the thermodynamics of nucleation follows a standard reasoning recalled hereafter (see e.g. [3],[8]) and nucleation could be defined as an adiabatic undrained crack propagation from 0 to $\ell^{*}$. However, adiabatic conditions lead to select the internal energy rather than the free energy for the formulation of the thermodynamics principles.

The starting point is the first principle: In adiabatic conditions, it states that the mechanical energy provided to the system $\Omega$ is transformed into internal energy $\mathscr{U}$ and kinetic energy $K$ :

$$
\dot{\mathscr{U}}+\dot{K}=\mathscr{P}_{e}
$$

For simplicity, we assume that the mechanical loading $\mathscr{L}$ is defined by body forces $\boldsymbol{F}$, prescribed surface forces $\lambda_{T}(t) \boldsymbol{T}^{0}$ on $\partial \Omega_{T}$, prescribed displacements $\lambda_{\xi}(t) \xi^{0}$ on $\partial \Omega_{\xi}$, where $\lambda_{T}(t)$ and $\lambda_{\xi}(t)$ are given scalar time functions while the boundary of the material system is $\partial \Omega=\partial \Omega_{\xi} \cup \partial \Omega_{T}$ :

$$
\mathscr{P}_{e}=\int_{\Omega} \boldsymbol{F} \cdot \dot{\boldsymbol{\xi}} d V+\lambda_{T}(t) \int_{\partial \Omega_{T}} \boldsymbol{T}^{0} \cdot \dot{\boldsymbol{\xi}} d S+\dot{\lambda}_{\xi}(t) \int_{\partial \Omega_{\xi}} \boldsymbol{T} \cdot \boldsymbol{\xi}^{0} d S
$$

The velocity is the sum of the contribution of the crack nucleation (under steady loading conditions) and that of the loading increment $\dot{\boldsymbol{\xi}}_{\mid \ell}$ (under constant crack length):

$$
\dot{\xi}=\frac{\partial \xi}{\partial \ell} \mid \mathscr{L}+\dot{\boldsymbol{\xi}}_{\mid \ell}
$$

Accordingly, the internal (resp. kinetic) energy is the sum of two terms, namely $(\partial \mathscr{U} / \partial \ell)_{\mid \mathscr{L}} \dot{\ell}\left(\operatorname{resp} .(\partial K / \partial \ell)_{\mid \mathscr{L}} \dot{\ell}\right)$ and $\dot{\mathscr{U}}_{\mid \ell}\left(\right.$ resp. $\left.\dot{K}_{\mid \ell}\right)$, which correspond to the contributions of crack nucleation and of the loading increment:

$$
\dot{\mathscr{U}}=\frac{\partial \mathscr{U}}{\partial \ell}{ }_{\mid \mathscr{L}} \dot{\ell}+\dot{\mathscr{U}}_{\mid \ell} \quad ; \quad \dot{K}=\frac{\partial K}{\partial \ell}{ }_{\mid \mathscr{L}} \dot{\ell}+\dot{K}_{\mid \ell}
$$

Similarly, the mechanical work is splitted into two terms $\mathscr{P}_{e \mid \mathscr{L}}$ and $\mathscr{P}_{e \mid \ell}$. Under steady loading conditions, the mechanical power is due to crack propagation only:

$$
\mathscr{P}_{e \mid \mathscr{L}}=\frac{\partial \Phi}{\partial \ell} \mid \mathscr{L} \quad \dot{\ell} \quad \text { with } \quad \Phi=\int_{\Omega} \boldsymbol{F} \cdot \boldsymbol{\xi} d V+\lambda_{T}(t) \int_{\partial \Omega_{T}} \boldsymbol{T}^{0} \cdot \boldsymbol{\xi} d S
$$

where $\Phi$ is the potential of the given loads. In turn, at constant crack length, the standard principle of virtual work readily yields:

$$
\mathscr{P}_{e \mid \ell}=\dot{K}_{\mid \ell}+\int_{\Omega} \boldsymbol{\sigma}: \dot{\varepsilon}_{\mid \ell} d V
$$


where $\boldsymbol{\sigma}$ and $\boldsymbol{\varepsilon}$ denote the local stress and strain fields. Let $u$ denote the volume density of internal energy. By nature, it is a function of the strain and of the entropy density $s$. However, at constant crack length, the evolution being reversible and adiabatic, it is also isentropic. The strain work $\boldsymbol{\sigma}: \dot{\boldsymbol{\varepsilon}}_{\mid \ell}$ is therefore equal to $\dot{u}_{\mid \ell}$ and the corresponding mechanical work reads:

$$
\mathscr{P}_{e \mid \ell}=\dot{K}_{\mid \ell}+\dot{\mathscr{U}}_{\mid \ell}
$$

Eventually, the combination of (2) and (3) yields:

$$
\frac{\partial}{\partial \ell} \mid \mathscr{L}(\mathscr{U}-\Phi+K)=0
$$

To this stage, it is important to observe that $\mathscr{U}$ is the total internal energy of the material system. Let $U$ and $U_{\mathscr{C}}$ respectively denote the internal energy of $\Omega(\ell)$ and of $\mathscr{C}(\ell)$. As an extensive quantity, the total internal energy $\mathscr{U}$ is the sum $U+U_{\mathscr{C}}$. (5) now takes the form:

$$
-\frac{\partial}{\partial \ell} \mid \mathscr{L}(U-\Phi+K)={\frac{\partial U_{\mathscr{C}}}{\partial \ell}}_{\mid \mathscr{L}}
$$

It remains to integrate the above equation over the process of nucleation which amounts to integrate w.r.t. $\ell$ between 0 and the length $\ell^{*}$ of the stable nucleated crack, under the constant loading $\mathscr{L}$. Let $U_{p}$ denote the potential energy $U-\Phi$. recalling that the initial state is an equilibrium (no kinetic energy), one obtains:

$$
U_{p}(0)-U_{p}\left(\ell^{*}\right)=U^{F D}+K\left(\ell^{*}\right)
$$

where $U^{F D}=U_{\mathscr{C}}\left(\ell^{*}\right)-U_{\mathscr{C}}(0)$ is the energy that was necessary for the nucleation. Due to adiabatic and undrained conditions, it is stored in the crack at the end of nucleation. This highlights the difference between the free and internal energies: the nucleated crack has no free energy but stores in adiabatic conditions the heat dissipated by the full damage process. (7) appears as the adiabatic counterpart of the isothermal energy balance derived in [14]. It states that the energy release of the bulk is the sum of the energy dissipated in the nucleation and of kinetic energy. The latter being a positive quantity, a necessary condition for nucleation reads:

$$
U_{p}(0)-U_{p}\left(\ell^{*}\right) \geq U^{F D}
$$

If we assume that the energy necessary for nucleation is proportional to the created crack surface $\ell^{*}\left(U^{F D}=\right.$ $\left.G_{c} \ell^{*}\right)$, the criterion for nucleation takes the form:

$$
G_{i n c}\left(\ell^{*}\right)=\frac{U_{p}(0)-U_{p}\left(\ell^{*}\right)}{\ell^{*}} \geq G_{c}
$$

Again, the above criterion and that derived in [14] look very much alike, the only difference being that the internal energy has replaced the free energy. In the use of (8), one should keep in mind its status of necessary condition for the nucleation of a crack with surface (resp. length) $\ell^{*}$.

By definition, the nucleation is the transition from the sound material to the cracked material, when a critical loading level is reached. By nature, it can be viewed as the propagation of a crack, starting from a length equal to 0 , but in a very short period of time. Therefore, as long as the length $\ell^{*}$ of the stable nucleated crack is not reached, the propagation condition holds in the classical form:

$$
G(\ell)=-\frac{\partial U_{p}}{\partial \ell}(\ell) \geq G_{c} \quad \text { for } \quad \ell \leq \ell^{*}
$$

The above inequality should be satisfied at least in a neighbourhood on the left of $\ell^{*}$. In contrast, once the critical length $\ell^{*}$ is reached, the condition for propagation should no more be violated:

$$
G\left(\ell^{*}\right)=G_{c} \quad \text { and } \quad G(\ell) \leq G_{c} \text { for } \ell \geq \ell^{*}
$$

The above inequality should be satisfied at least in a neighbourhood on the right of $\ell^{*}$. Observing that $G_{i n c}\left(0^{+}\right)=0$ and $G_{i n c}\left(\ell^{*}\right)=G_{c}$, it is expected that $G_{i n c}(\ell)$ is an increasing function on the interval $\left[0, \ell^{*}\right]$. Accordingly, $d G_{i n c} / d \ell(\ell) \geq 0$ :

$$
\left(\forall \ell \leq \ell^{*}\right) \quad \frac{d G_{i n c}}{d \ell}(\ell)=\frac{G(\ell)-G_{i n c}(\ell)}{\ell} \geq 0 \Rightarrow G\left(\ell^{*}\right) \geq G_{i n c}\left(\ell^{*}\right)
$$


Putting together (8), (10) and (11), it is concluded that

$$
G\left(\ell^{*}\right)=G_{i n c}\left(\ell^{*}\right)=G_{c}
$$

Therefore, (11) shows that $G_{i n c}^{\prime}\left(\ell^{*}\right)=0$. This means that a stable nucleation corresponds to a maximum of the function $G_{i n c}(\ell)$. The above reasoning is summarized by figure 2 .

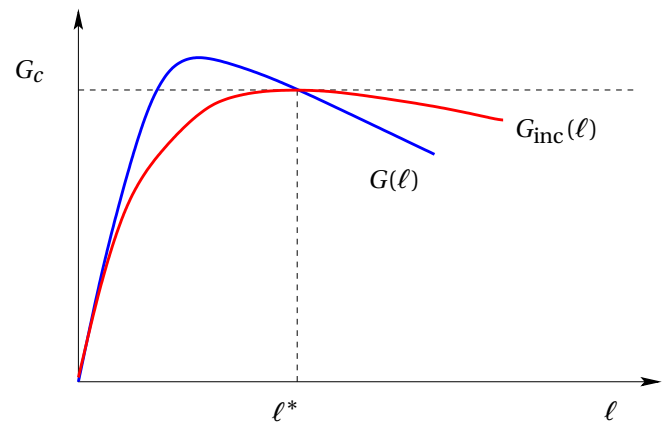

Fig. 2 Characterization of the nucleated crack length $\ell^{*}$ : differential and incremental rates of energy release.

In practice, once the orientation of the crack is chosen, there are two unknowns, namely the loading level and the critical length $\ell^{*}$. They are determined by the conditions

$$
G_{i n c}\left(\ell^{*}\right)=G_{c} \quad ; \quad \frac{d G_{i n c}}{d \ell}\left(\ell^{*}\right)=0
$$

This criterion has a general validity, but its practical implementation requires to make an assumption on the fracture mechanism in order to derive the function $G_{i n c}(\ell)$. In other words, it is necessary to specify the mode of fracturing. Nevertheless, it is possible to discriminate between several possible mechanisms.

According to the definition (8), the method only requires the numerical determination of the potential energy of the initial state as well as that of the perturbated state. The underlying theoretical argument is the theorem of the minimum potential energy. Let $\xi^{0}$ denote the displacement field prior to nucleation and $\delta \xi$ denote an arbitrary kinematically admissible perturbation. The principle of minimum potential energy states that

$$
U_{p}(\ell)=\min _{\delta \xi \mathrm{k} . \mathrm{a} .} U_{p}^{\ell}\left(\boldsymbol{\xi}^{0}+\delta \boldsymbol{\xi}\right)
$$

In (13), $U_{p}^{\ell}$ denote the potential energy function which operates on the space of virtual displacement fields, while $U_{p}(\ell)$ represents the potential energy of the elastic solution for the geometry defined by $\ell$. For given crack length $\ell$ and orientation, the variational problem (13) can be adressed by means of a finite element computational technique. Alternatively, it is possible to determine an upper bound value of $U_{p}(\ell)$ while restricting to a small number of optimization parameters in (13). In both cases, for a given value of $\ell, G_{i n c}(\ell)$ is the solution to the following variational problem:

$$
G_{i n c}(\ell)=\max _{\delta \xi \mathrm{k} . \mathrm{a} .} \frac{U_{p}^{0}\left(\xi^{0}\right)-U_{p}^{\ell}\left(\xi^{0}+\delta \xi\right)}{\ell}
$$

The second step consists in the determination of the maximum, w.r.t. $\ell$, of the function $G_{i n c}(\ell)$. The practical implementation of this reasoning will be presented at section 4.4 .

Akin to the potential energy, $G_{i n c}(\ell)$ is a quadratic function of the loading parameter denoted formally as Q:

$$
G_{i n c}(\ell)=Q^{2} \tilde{G}_{i n c}(\ell)
$$

The loading level $Q^{*}$ that triggers nucleation is derived from (12):

$$
Q^{*}=\sqrt{\frac{G_{c}}{\max _{\ell} \tilde{G}_{i n c}(\ell)}}
$$


Note that any approximate solution of the variational problem (14) which restricts to a subset of the space of virtual displacements $\delta \xi$ will yield a lower bound estimate $\tilde{G}_{i n c}^{\text {num }}(\ell) \leq \tilde{G}_{i n c}(\ell)$. The loading level $\sqrt{G_{c} / \tilde{G}_{i n c}^{\text {num }}\left(\ell^{*}\right)}$ predicted by the nucleation criterion (12) will therefore overestimate the actual failure loading level.

\section{The saturated porous medium}

The assumption of undrained local behavior amounts to neglect any fluid flow. At first sight, this means that the porous material can be regarded as a particular type of composite material. This is the point of view that was adopted in section 2 in which all energy quantities refer to this solid-fluid mixture. The purpose of the present section is to go a step further in the discussion on the influence of a pore fluid in the nucleation of cracks. The focus is put on the poromechanical coupling. For the sake of simplicity, in this section only, the temperature change is neglected. Prior to the instant of nucleation, the physical state of the fluid is assumed to be the liquid one.

In order to get a closer insight in the nucleation process, the crack is viewed as a three-dimensional material system, as already mentionned at section 2. In order to preserve the possibility of deriving closed-form expressions, we restrict to the spheroidal crack model embedded in an infinite isotropic porous medium $\Omega$, with a uniform asymptotic strain state $\boldsymbol{E}$. The region outside the spheroid $\mathscr{E}$ has a poroelastic behavior. The stress $\boldsymbol{\sigma}^{\mathscr{E}}$, strain $\boldsymbol{\varepsilon}^{\mathscr{E}}$, the pore pressure $p^{\mathscr{E}}$ and the damage $d$ are uniform in the spheroid $\mathscr{E}$. Note that the reference state for the strain is the natural state (stress and pressure equal to 0 ).

As expected, crack nucleation in mode II induced by shear loading does not affect the pore pressure, which means that there is no poromechanical coupling in this loading case [22]. In contrast, at the scale of the spheroidal crack model, crack nucleation in mode I is expected to induce positive strain in the direction normal to the crack, so as to increase the crack thickness. In undrained condition, this tendency to open the crack is expected to produce a pressure decrease. As long as the fluid pressure remains above the saturation vapour pressure, it is reasonable to assume a constant compressibility of the liquid. However, if the pressure drops until it reaches saturation vapour pressure (see figure 3), the compressibility suddenly increases by several orders of magnitude. For a full understanding of the nucleation process, a prediction of pore pressure change is therefore necessary. For this purpose, a theory of poroelasticity with damage is now considered.

\subsection{Elements of damage theory for poroelastic media}

Let $\phi$ denote the lagrangian porosity defined as the pore volume in a representative elementary volume (r.e.v.) normalized by the reference volume of the latter. $\phi_{0}$ is the initial porosity. We also denote by $\psi$ the (lagrangian) volume density of free energy in the solid phase of the r.e.v.. The rate of dissipated energy per unit volume $D$ in an isothermal evolution reads [4]:

$$
D=\boldsymbol{\sigma}: \dot{\boldsymbol{\varepsilon}}+p \dot{\phi}-\dot{\psi}
$$

In order to adopt the strain $\varepsilon$ and the fluid pressure $p$ as state variables, it is convenient to introduce the potential energy density $\psi^{*}=\psi-p\left(\phi-\phi_{0}\right)$ with which the dissipation also takes the form:

$$
D=\boldsymbol{\sigma}: \dot{\boldsymbol{\varepsilon}}-\dot{\psi}^{*}-\dot{p}\left(\phi-\phi_{0}\right)
$$

Accordingly, $\psi^{*}$ is a function of $\boldsymbol{\varepsilon}$ and $p$ and of the internal variable $d$. The state equations read:

$$
\boldsymbol{\sigma}=\frac{\partial \psi^{*}}{\partial \boldsymbol{\varepsilon}} \quad ; \quad \phi-\phi_{0}=-\frac{\partial \psi^{*}}{\partial p}
$$

and the dissipation reduces to:

$$
D=-\frac{\partial \psi^{*}}{\partial d} \dot{d}
$$


The thermodynamic force controlling damage appears to be $-\partial \psi^{*} / \partial d$. Assuming that there exists a material constant $g_{c}$ such that dissipation reads $g_{c} \dot{d}$, the local damage criterion takes the form:

$$
-\frac{\partial \psi^{*}}{\partial d}=g_{c}
$$

The material constant $g_{c}$ is the three-dimensional counterpart of the critical energy $G_{c}$ appearing in (12). In linear poroelasticity, $\psi^{*}$ is a quadratic polynomial:

$$
\psi^{*}=\frac{1}{2} \varepsilon: \mathbb{C}_{d r}: \boldsymbol{\varepsilon}-p \boldsymbol{B}: \boldsymbol{\varepsilon}-\frac{p^{2}}{2 N}
$$

Note that the validity of this expression requires that the reference state is the natural one (free of stress and fluid pressure). Through the damage process, the drained elastic stiffness tensor $\mathbb{C}_{d r}$ is a function of the damage parameter $d$. In general, this also holds for the Biot tensor $\boldsymbol{B}$ since it is related to the elastic stiffness tensor of the solid phase $\mathbb{C}_{s}$ and to the effective drained stiffness tensor $\mathbb{C}_{d r}$ by:

$$
\boldsymbol{B}=\boldsymbol{\delta}:\left(\mathbb{\square}-\mathbb{C}_{s}^{-1}: \mathbb{C}_{d r}\right)
$$

where $\boldsymbol{\delta}$ (resp. ) is the second-order (resp. symmetrized fourth-order) unit tensor. The first state equation reads:

$$
\boldsymbol{\sigma}=\mathbb{C}_{d r}(d): \boldsymbol{\varepsilon}-p \boldsymbol{B}(d)
$$

As opposed to $\mathbb{C}_{d r}$, note that $\mathbb{C}_{s}$ is not affected by the damage process and can be regarded as an invariable material constant. The Biot modulus at constant porosity $N$ is related to $\boldsymbol{B}$ and $\mathbb{C}_{s}$ :

$$
\frac{1}{N}=\boldsymbol{\delta}: \mathbb{C}_{s}^{-1}:\left(\boldsymbol{B}-\phi_{0} \boldsymbol{\delta}\right)
$$

It is affected by the damage process through its link with the Biot tensor. The damage criterion (21) is a quadratic function of $\varepsilon$ and $p$ :

$$
-\frac{\partial \psi^{*}}{\partial d}=-\frac{1}{2} \varepsilon: \frac{\partial}{\partial d}\left(\mathbb{C}_{d r}\right): \varepsilon+p \frac{\partial}{\partial d}(\boldsymbol{B}): \varepsilon+\frac{p^{2}}{2} \frac{\partial}{\partial d}\left(\frac{1}{N}\right)=g_{c}
$$

Using (23) and (25), the thermodynamic force of damage proves to depend on $\varepsilon$ and $p$ through an effective strain $\boldsymbol{\varepsilon}^{\prime}=\boldsymbol{\varepsilon}+p \mathbb{C}_{s}^{-1}: \boldsymbol{\delta}$ :

$$
-\frac{\partial \psi^{*}}{\partial d}=-\frac{1}{2}\left(\boldsymbol{\varepsilon}+p \mathbb{C}_{s}^{-1}: \boldsymbol{\delta}\right): \frac{\partial}{\partial d}\left(\mathbb{C}_{d r}\right):\left(\boldsymbol{\varepsilon}+p \mathbb{C}_{s}^{-1}: \boldsymbol{\delta}\right)=g_{c}
$$

Using (22) in (19) together with (23) yields:

$$
\boldsymbol{\sigma}+p \boldsymbol{\delta}=\mathbb{C}_{d r}:\left(\boldsymbol{\varepsilon}+p \mathbb{C}_{s}^{-1}: \boldsymbol{\delta}\right)
$$

Eventually, it appears that the damage criterion is controlled by the so-called Terzaghi effective stress tensor $\boldsymbol{\sigma}+p \boldsymbol{\delta}:$

$$
\frac{1}{2}(\boldsymbol{\sigma}+p \boldsymbol{\delta}): \frac{\partial}{\partial d}\left(\mathbb{C}_{d r}^{-1}\right):(\boldsymbol{\sigma}+p \boldsymbol{\delta})=g_{c}
$$

In the following, we restrict for simplicity to the limit case $:\left|\boldsymbol{\delta}: \mathbb{C}_{s}{ }^{-1}: \mathbb{C}_{d r}\right| \ll 1$. In short, this means that the compressibility of the solid phase is low as compared to that of the porous medium. This implies that $\boldsymbol{B} \rightarrow \boldsymbol{\delta}$. Besides, from (27), it is found that the damage criterion can be put in the form of a quadratic polynomial on $\varepsilon$ alone:

$$
-\frac{\partial \psi^{*}}{\partial d}=-\frac{1}{2} \varepsilon: \frac{\partial}{\partial d}\left(\mathbb{C}_{d r}\right): \varepsilon=g_{c}
$$




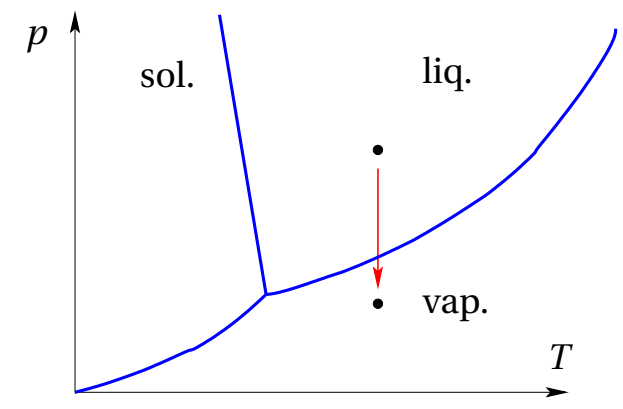

Fig. 3 Phase diagram of water

\subsection{Modeling the damage process}

Let $\boldsymbol{E}^{e l}$ and $p_{0}$ denote the strain level and the pore fluid pressure at the onset of damage. Prior to damage, the strain field and the pore pressure are uniform throughout $\Omega$. As already stated, it is assumed that the damage process that represents nucleation takes place in undrained conditions. During the damage process in the damaged zone $\mathscr{E}$, the stress, the strain and the fluid pressure are related by the state equation (24) $(\boldsymbol{B} \rightarrow \boldsymbol{\delta})$ :

$$
\boldsymbol{\sigma}^{\mathscr{E}}=\mathbb{C}_{d r}(d): \boldsymbol{\varepsilon}^{\mathscr{E}}-p^{\mathscr{E}} \boldsymbol{\delta}
$$

During an undrained (isothermal) evolution, the pressure change is controlled by the undrained constraint:

$$
0=\boldsymbol{\delta}:\left(\boldsymbol{\varepsilon}-\boldsymbol{E}^{e l}\right)+\frac{p-p_{0}}{M} \quad \text { with } \quad \frac{1}{M}=\frac{1-\phi_{0}}{K_{s}}+\frac{\phi_{0}}{K_{f}}=\overline{1 / K}
$$

in which $K_{f}$ and $K_{s}$ are the compression modulii of the fluid and solid phases respectively. This equation holds in the damaged zone with $\boldsymbol{\varepsilon}=\boldsymbol{\varepsilon}^{\mathscr{E}}$ and $p=p^{\mathscr{E}}$ as well as outside for appropriate values of $\boldsymbol{\varepsilon}$ and $p$. The above state equation therefore yields:

$$
\boldsymbol{z} \in \mathscr{E}: \quad \boldsymbol{\sigma}^{\mathscr{E}}=\mathbb{C}_{u}(d): \boldsymbol{\varepsilon}^{\mathscr{E}}+\boldsymbol{\tau}_{0} \quad \text { with } \quad \boldsymbol{\tau}_{0}=-p_{0} \boldsymbol{\delta}-M \boldsymbol{\delta} \otimes \boldsymbol{\delta}: \boldsymbol{E}^{e l}
$$

in which the undrained stiffness tensor reads:

$$
\mathbb{C}_{u}(d)=\mathbb{C}_{d r}(d)+M \boldsymbol{\delta} \otimes \boldsymbol{\delta}
$$

Note that the Biot modulus in undrained conditions ${ }^{1} M$ is not affected by damage. We herein consider a class of isotropic damage models in which $\mathbb{C}_{d r}(d)=3 k^{d r}(d) \mathbb{J}+2 \mu(d) \mathbb{K}$ with:

$$
\frac{k^{d r}(d)}{k_{0}^{d r}}=\frac{\mu(d)}{\mu_{0}}=f(d) \quad \text { with } \quad f(d)=\frac{1-Q d}{1+Q^{\prime} d}
$$

with $Q, Q^{\prime}>0$. For practical applications and numerical simulations, $f(d)$ will be chosen as the ratio $k^{d r}(d) / k_{0}^{d r}$ predicted by the Ponte-Castaneda-Willis homogenization scheme [13] for an isotropic solid damaged by a network of microcracks with isotropic distribution of orientations:

$$
Q=\frac{32}{27}\left(1+v_{0}^{d r}\right) \quad ; \quad Q^{\prime}=\frac{16}{27} \frac{\left(1+v_{0}^{d r}\right)^{2}}{1-2 v_{0}^{d r}}
$$

The very important feature in (33) is that the undrained bulk modulus $k^{u}$ now comprises a component that is not subjected to damage:

$$
k^{u}(d)=k^{d r}(d)+M
$$

Out of the domain $\mathscr{E}$, the state equation involves the sound undrained stiffness tensor $\mathbb{C}_{u}^{0}=\mathbb{C}_{u}(0)$ :

$$
z \in \Omega \backslash \mathscr{E}: \quad \boldsymbol{\sigma}=\mathbb{C}_{u}^{0}: \boldsymbol{\varepsilon}+\boldsymbol{\tau}_{0}
$$

1 Note that $M=N$ for an incompressible fluid $\left(K_{f} \rightarrow \infty\right)$. 
Since $\boldsymbol{\tau}_{0}$ is a constant, (32) shows that the damage process in an undrained material is formally identical to that of a non porous material. At the strain level when the damage process is initiated in $\mathscr{E}$, the strain field is still uniform throughout $\Omega$ so that the damage criterion (30) reads

$$
-\frac{1}{2} \boldsymbol{E}^{e l}: \frac{\partial}{\partial d}\left(\mathbb{C}_{d r}\right): \boldsymbol{E}^{e l}=g_{c}
$$

which yields the elastic threshold $\boldsymbol{E}^{e l}=E^{e l} \boldsymbol{\delta}$ in the form:

$$
E^{e l}=\frac{1}{3} \sqrt{-\frac{2 g_{c}}{\partial k^{d r} / \partial d(0)}} \quad \text { with } \quad \frac{\partial k^{d r}}{\partial d}(0)=-\left(Q+Q^{\prime}\right) k_{0}^{d r}
$$

Beyond the threshold $E^{e l}$, we need to determine 3 unknowns, namely the strain tensor $\boldsymbol{\varepsilon}^{\mathscr{E}}$, the damage level $d$ and the fluid pressure $p^{\mathscr{E}}$. The first equation is provided by the damage criterion:

$$
-\frac{1}{2} \boldsymbol{\varepsilon}^{\mathscr{E}}: \frac{\partial}{\partial d}\left(\mathbb{C}_{d r}\right): \boldsymbol{\varepsilon}^{\mathscr{E}}=g_{c}
$$

We then observe from (32) and (35) that $\boldsymbol{\varepsilon}^{\mathscr{E}}$ is the solution of an Eshelby problem (see (90)):

$$
\boldsymbol{\varepsilon}^{\mathscr{E}}=\left(\mathbb{q}+\mathbb{P}_{0}^{u}:\left(\mathbb{C}_{u}(d)-\mathbb{C}_{u}^{0}\right)\right)^{-1}: \boldsymbol{E}
$$

Eliminating $\boldsymbol{\varepsilon}^{\mathscr{E}}$, these two equations can be solved with respect to $\boldsymbol{E}$ and $d$. Finally, the fluid pressure is derived from the undrained constraint (31):

$$
p^{\mathscr{E}}=p_{0}-M \boldsymbol{\delta}:\left(\boldsymbol{\varepsilon}^{\mathscr{E}}-\boldsymbol{E}^{e l}\right)
$$

The numerical simulations require numerical values for the ratios $g_{c} / Y_{0}^{d r}$ and $M / Y_{0}^{d r}$ ( $Y_{0}^{d r}$ is the drained

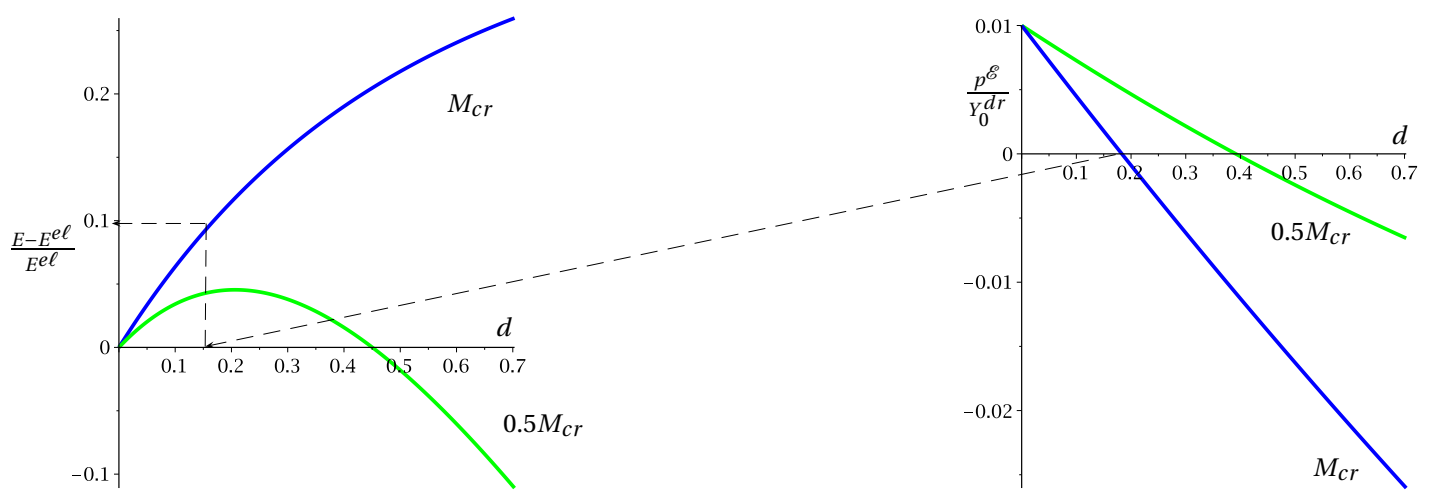

Fig. 4 Asymptotic strain $E$ and crack fluid pressure $p^{\mathscr{E}}$ as functions of the damage parameter $d . g_{c} / Y_{0}^{d r}=10^{-3}, v_{0}^{d r}=0.2, p_{0} / Y_{0}^{d r}=$ $10^{-2}$

Young modulus of the sound material), and for its Poisson coefficient $v_{0}^{d r}$. For further use, we introduce the critical Biot modulus $M_{c r}$ defined as:

$$
M_{c r}=\left(k_{0}^{d r}+\frac{4}{3} \mu_{0}^{d r}\right) \frac{Q}{Q^{\prime}}=2 Y_{0}^{d r} \frac{\left(1-v_{0}^{d r}\right)}{\left(1+v_{0}^{d r}\right)^{2}}
$$

Typical results are presented at figure 4 for two values of $M$, respectively $M_{c r}$ and $0.5 M_{c r}$. On the left (resp. on the right), the loading level $E$ (resp. the pore pressure) is presented as a function of damage. For $M=0.5 M_{c r}$, the asymptotic strain $E$ is not a monotonic function of the damage parameter. This indicates that the response of the system is not stable. In contrast, for $M=M_{c r}$, the asymptotic strain $E$ is a strictly increasing function of the damage parameter. This suggests that the response could be stable. Indeed, if shear modes are disregarded, the condition of stability provided by Hill's criterion was shown to be satisfied 
if $M \geq M_{c r}$ [22].

The central role of the Biot modulus in the discussion on the stability of the damage process deserves that its micromechanical interpretation be recalled (see (31)). In water saturated rocks, the property $K_{s} \gg K_{f}$ allows to approximate $M$ by the ratio $K_{f} / \phi_{0}$, so that the numerical value of $M$ is related to the fluid compressibility. As the stable damage process goes on, the pore pressure progressively decreases. When the pressure reaches the saturation vapour pressure ( 0 in practice), the liquid is partly vaporized which amounts to a dramatic drop of the Biot modulus $M$. The brittle process leading to nucleation is then triggered.

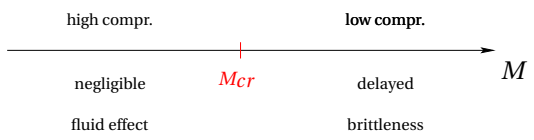

Fig. 5 The role of $M$ on the stability of the damage process

The conclusions of the discussion can be summarized by the scheme of figure 5. If $M<M_{c r}$ (high fluid compressibility), a non stable damage process (modelled according to the principles of the damage theory of section 3.1) is obtained. This strongly suggests that a brittle failure mechanism akin to the non porous case takes place at or shortly after the initiation of damage $\left(E=E^{e l}\right)$. In this case, the fluid role is negligible. In contrast, if $M>M_{c r}$, the damage process is stable as long as the condition for vaporization is not reached, that is as long as the liquid pressure $p^{\mathscr{E}}$ is greater than the saturation vapour pressure $p_{v}^{\text {sat }}$. The condition for physical phase change $p^{\mathscr{E}}=p_{v}^{\text {sat }}$ corresponds to a second threshold $E^{*}>E^{e l}$. At this instant, a brittle failure mechanism is possible due to the dramatic increase of fluid compressibility. Nevertheless, the existence of a stable loading interval $E \in\left[E^{e l}, E^{*}\right]$ represents a delay in the material brittleness (see figure 6).

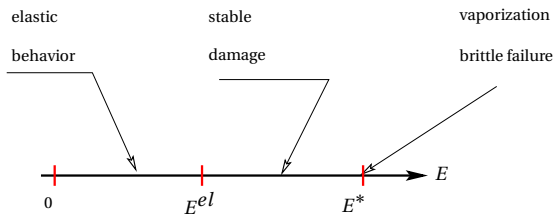

Fig. 6 Delayed brittle response in the case $M>M_{c r}$

\section{Failure Mechanism at the wellbore}

The wellbore in a medium subjected to remote stresses is now considered in a plane strain model. A circular hole (radius $R$ ) centered at the origin $O$ of the $O x y$ plane is surrounded by an infinite linear elastic isotropic medium (Young modulus $Y$, Poisson ratio $v$, elastic stiffness tensor $\mathbb{C}$ ). The elastic properties are implicitely the undrained adiabatic ones. However, the delay in brittleness discussed in the former section is now neglected.

Far from the hole, the remote state of stress is characterized by the principal stresses $\sigma_{x x}^{\infty}, \sigma_{y y}^{\infty}<0$ :

$$
\boldsymbol{\sigma}^{\infty}=\sigma_{x x}^{\infty} \boldsymbol{e}_{x} \otimes \boldsymbol{e}_{x}+\sigma_{y y}^{\infty} \boldsymbol{e}_{y} \otimes \boldsymbol{e}_{y}+\sigma_{z z}^{\infty} \boldsymbol{e}_{z} \otimes \boldsymbol{e}_{z} \quad \text { with } \quad \sigma_{z z}^{\infty}=v\left(\sigma_{x x}^{\infty}+\sigma_{y y}^{\infty}\right)
$$

(38) implicitely assumes that the axis of the wellbore is also a principal direction of the stress tensor. The present study is restricted to this assumption. The more general situation where the wellbore axis is inclined with respect to the principal direction will be considered in a forthcoming paper.

Taking the natural state as reference, the remote state of strain is:

$$
\varepsilon^{\infty}=\mathbb{C}^{-1}: \boldsymbol{\sigma}^{\infty}
$$

As regards the asymptotic boundary conditions, stresses or displacements are a priori possible. This choice affects the definition of the potential energy of the problem. 
In order to simulate the drilling process during which the contact force acting on the wellbore wall (with outward unit normal $\boldsymbol{n}=-\boldsymbol{e}_{r}$ ) decreases from $\boldsymbol{\sigma}^{\infty} \cdot \boldsymbol{n}$ to 0 , the stress vector acting on the wall is defined by $-\eta(t) \boldsymbol{\sigma}^{\infty} \cdot \boldsymbol{e}_{r}$ where $\eta(t) \in[0,1]$ is a decreasing function of time accounting for the progressive drilling process $(\eta(0)=1)$. Clearly, intermediate values of $\eta(t) \in] 0,1$ [ are rather of academical interest. In engineering practice, the value $\eta=0$ aims at modelling complete removal of the initial material. The corresponding mechanical loading must be considered for the sake of security. Indeed, it seems dangerous to assume that the initial pressure acting on the wellbore wall can be instantaneously replaced by a mud pressure, even if the time interval in between is short. Besides, apart from application to the wellbore, the situation corresponding to $\eta=0$ is also useful for the stability analysis of unlined tunnels.

In the present work, the most severe loading corresponds to $\eta=0$. This means that no mud pressure is accounted for. This could be done easily, and is by no means due to a limitation of the methodology, but goes beyond the scope of the paper.
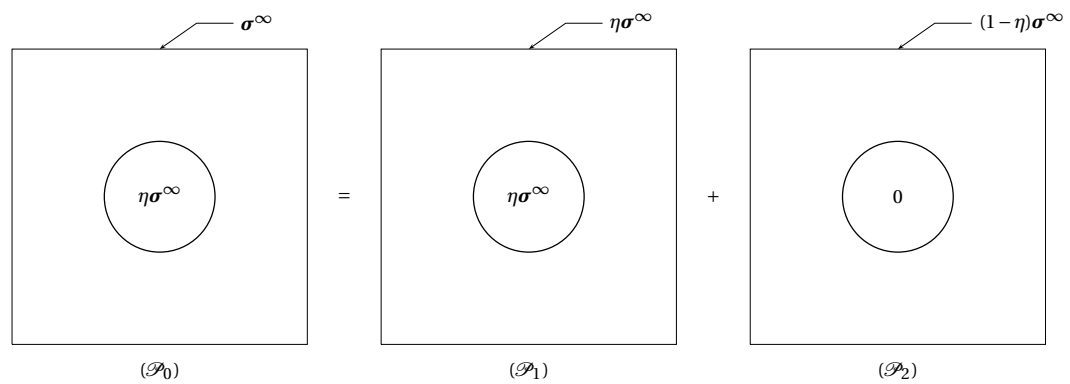

Fig. 7 Problem decomposition

\section{1 determination of the uncracked stress field}

The stress field in the uncracked configuration, for a given value of $\eta$, may be determined as the superposition of the solutions of two auxiliary problems (Fig. 7).

For auxiliary problem $\left(\mathscr{P}_{1}\right)$, the solution is obviously the uniform state of stress $\boldsymbol{\sigma}^{(1)}=\eta \boldsymbol{\sigma}^{\infty}$. For practical reasons, it is useful to introduce $\sigma_{m}^{\infty}=\left(\sigma_{x x}^{\infty}+\sigma_{y y}^{\infty}\right) / 2$ and $\sigma_{d}^{\infty}=\left(\sigma_{x x}^{\infty}-\sigma_{y y}^{\infty}\right) / 2$, the in-plane mean and deviatoric stresses respectively. Considering the cylindrical frame $\left(\boldsymbol{e}_{r}, \boldsymbol{e}_{\theta}, \boldsymbol{e}_{z}\right)$, Eq. (38) takes the form:

$$
\sigma_{r r}^{\infty}=\sigma_{m}^{\infty}+\sigma_{d}^{\infty} \cos 2 \theta \quad ; \quad \sigma_{\theta \theta}^{\infty}=\sigma_{m}^{\infty}-\sigma_{d}^{\infty} \cos 2 \theta \quad ; \quad \sigma_{r \theta}^{\infty}=-\sigma_{d}^{\infty} \sin 2 \theta
$$

The solution of auxiliary problem $\left(\mathscr{P}_{2}\right)$ reads ([31]):

$$
\begin{aligned}
& \sigma_{r r}^{(2)}=(1-\eta)\left(\sigma_{m}^{\infty}\left(1-\frac{R^{2}}{r^{2}}\right)+\sigma_{d}^{\infty}\left(1-\frac{4 R^{2}}{r^{2}}+\frac{3 R^{4}}{r^{4}}\right) \cos 2 \theta\right) \\
& \sigma_{\theta \theta}^{(2)}=(1-\eta)\left(\sigma_{m}^{\infty}\left(1+\frac{R^{2}}{r^{2}}\right)-\sigma_{d}^{\infty}\left(1+\frac{3 R^{4}}{r^{4}}\right) \cos 2 \theta\right) \\
& \sigma_{r \theta}^{(2)}=-(1-\eta) \sigma_{d}^{\infty}\left(1+\frac{2 R^{2}}{r^{2}}-\frac{3 R^{4}}{r^{4}}\right) \sin 2 \theta
\end{aligned}
$$

The state of stress in the uncracked configuration defined as $\boldsymbol{\sigma}^{0}=\boldsymbol{\sigma}^{(1)}+\boldsymbol{\sigma}^{(2)}$ is therefore:

$$
\begin{aligned}
& \sigma_{r r}^{0}=\sigma_{m}^{\infty}\left(1-(1-\eta) \frac{R^{2}}{r^{2}}\right)+\sigma_{d}^{\infty}\left(1-(1-\eta)\left(\frac{4 R^{2}}{r^{2}}-\frac{3 R^{4}}{r^{4}}\right)\right) \cos 2 \theta \\
& \sigma_{\theta \theta}^{0}=\sigma_{m}^{\infty}\left(1+(1-\eta) \frac{R^{2}}{r^{2}}\right)-\sigma_{d}^{\infty}\left(1+3(1-\eta) \frac{R^{4}}{r^{4}}\right) \cos 2 \theta \\
& \sigma_{r \theta}^{0}=-\sigma_{d}^{\infty}\left(1+(1-\eta)\left(\frac{2 R^{2}}{r^{2}}-\frac{3 R^{4}}{r^{4}}\right)\right) \sin 2 \theta
\end{aligned}
$$


Note that the initial state of stress that has been derived assumes that the material around the wellbore is homogeneous. Practically, this eliminates the situation of pre-existing flaws or inhomogeneities. This is not a limitation of the proposed method, but goes beyond the scope of the present paper.

Section 2 has provided a criterion for crack nucleation. Among various assumptions that can be made concerning the crack mode and the crack geometry, the mechanism that is likely to take place is the one that minimizes the required loading level. Three different models are presented hereafter in sections $4.2,4.3$ and 4.4 respectively.

\subsection{Mode I cracks induced by uniaxial compression}

In this section, we focus on the particular case of tensile cracks induced in uniaxial compression $\left(\sigma_{y y}^{\infty}<0\right.$, figure 1 , left) when no stresses act on the cavity wall $(\eta=0)$. The possibility of nucleation of a tensile crack is supported by the analysis of the stress field in the vicinity of the borehole, along the $O y$-axis $(\theta=\pi / 2$ or $3 \pi / 2$ ). From (42), it is found that:

$$
\sigma_{x x}(0, y)=\frac{1}{2} \sigma_{y y}^{\infty} \frac{R^{2}}{r^{2}}\left(1-3 \frac{R^{2}}{r^{2}}\right) \quad \text { with } r=|y|
$$

It appears that this loading induces a tensile stress for $r \in[R, R \sqrt{3}]$. For a sufficiently high value of $\sigma_{y y}^{\infty}$, the nucleation of a crack in mode I is therefore expected in this region of space. We have extensively commented on the link between nucleation and damage in section 2 . It is therefore interesting to discuss whether this location could be confirmed by means of a damage criterion.

\subsubsection{Damage initiation}

The reference state being the natural state, the criterion for damage initiation during an adiabatic loading reads [16]:

$$
-\frac{\partial u}{\partial d}=-\frac{1}{2} \varepsilon: \frac{\partial \mathbb{C}}{\partial d}: \varepsilon=g_{c}
$$

In the above equation, it is emphasized that $\mathbb{C}$ denotes the adiabatic stiffness tensor while $u$ denotes the volume density of internal energy of the solid-fluid mixture. To begin with, let us resort to an isotropic model in which damage is attributed to microcracks of random orientation. For simplicity, it is first assumed that damage is controlled by a single scalar parameter $d$. The bulk and shear modulii $k(d)$ and $\mu(d)$ follow the same damage law:

$$
\frac{k(d)}{k_{0}}=\frac{\mu(d)}{\mu_{0}}=f(d)
$$

in which $f(d)$ is a decreasing function of $d$. This kind of damage law can be supported by a micromechanics modelling of the effect of microcracks on the effective stiffness in which $d$ is related to the crack density parameter (see e.g. [13]). To this stage, it is unnecessary to specify the function $f(d)$. Using 42 with $\theta=\pi / 2$, it is found that $-\partial u / \partial d$ is a function of $\rho=r / R$ :

$$
-\frac{\partial u}{\partial d}(\rho)=\frac{1+v}{2} \frac{\left|f^{\prime}(d)\right|}{f(d)^{2}} \frac{\sigma_{y y}^{\infty}}{Y}\left(1-v+\frac{4 v-5}{\rho^{2}}+\frac{19 / 2-4 v}{\rho^{4}}-\frac{9}{\rho^{6}}+\frac{9}{2 \rho^{8}}\right)
$$

The normalized function by the value reached at $r=R$ is plotted at figure 8 . Unexpectedly, the energy release exhibits a minimum in the region of tensile stress. The damage criterion is obviously not consistent with the physical expectation of crack nucleation. This shortcoming is attributed to the unrealistic assumption of isotropic damage, at least along the $O y$ axis in the vicinity of the wellbore, where the principle stresses exhibit opposite signs. The idea is that the tensile stress $\sigma_{x x}$ induces the nucleation of microcracks that are oriented parallel to the $O y$ axis. The nucleation of the macrocrack along this axis can be viewed as the final stage of this process.

This anisotropy of the expected damage can be easily taken into account by means of an homogenization scheme. The simplest approach consists in using the dilute scheme. Assuming a density $n$ of parallel cracks with half-length $a$, the crack density parameter is $d=\pi n^{2}$ and the effective stiffness reads (see e.g. [3]):

$$
\mathbb{C}(d)=\mathbb{C}(0):(\mathbb{Q}-d \mathbb{\mathbb { T }})
$$




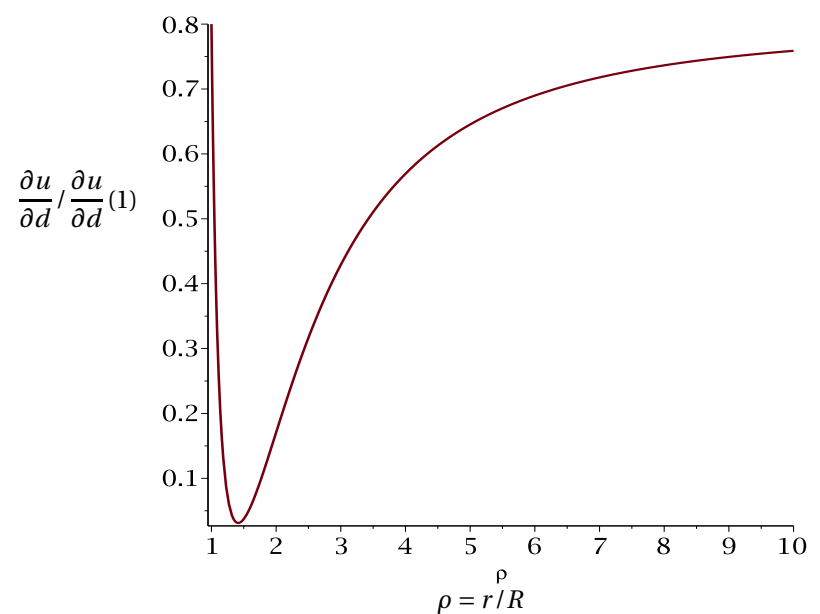

Fig. 8 Normalized $-\partial u / \partial d$ along $O y$ as a function of $\rho=r / R$ for a uniaxial compressive remote stress $\sigma_{y y}^{\infty}$ : Isotropic damage.

where

$$
T_{x x y y}=\frac{2 v(1-v)}{1-2 v} \quad ; \quad T_{x x x x}=\frac{2(1-v)^{2}}{1-2 v} \quad ; \quad T_{x y x y}=\frac{1-v}{2}
$$

The rate of energy release damage initiation criterion 44 now reads:

$$
-\frac{\partial u}{\partial d}(\rho)=\frac{\left(1-v^{2}\right)}{4} \frac{\sigma_{y y}^{\infty}}{Y} \frac{\left(3-\rho^{2}\right)^{2}}{\rho^{8}}
$$

The normalized function by the value reached at $r=R$ is plotted at figure 9. Clearly enough, the corre-

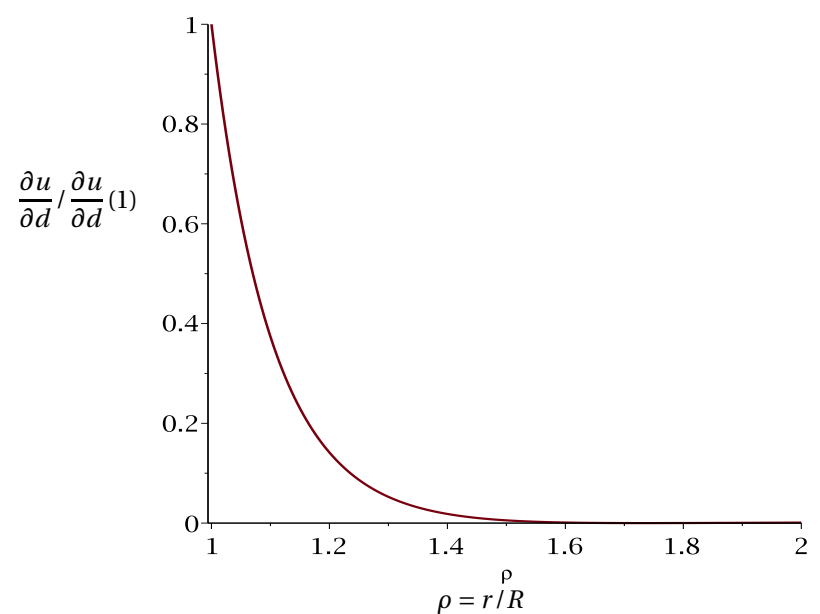

Fig. 9 Normalized $-\partial u / \partial d$ along $O y$ as a function of $\rho=r / R$ for a uniaxial compressive remote stress $\sigma_{y y}^{\infty}$ : Anisotropic damage.

sponding criterion for damage initiation is now in full agreement with the physical expectation based on the existence of a tensile stress $\sigma_{x x}$ along $O y$. This emphasizes that anisotropy of damage plays an essential role in view of predicting nucleation. It seems that this question remains quite open as far as the variational approach of crack nucleation is concerned.

\subsubsection{Tensile crack nucleation}

The implementation of the criterion of nucleation presented at section 2 is particularly simplified when an analytical expression of the finite increase rate $G_{i n c}(\ell)$ is available. In the present case of a tensile crack 
along the $O y$ axis as depicted at figure 1 (left), the stress intensity factor for a given remote stress state is wellknown in the literature [28]:

$$
K_{I}(\ell) \approx-\frac{1.1 \sqrt{\ell / R}}{(1+\ell / R)^{3.3}} \sigma_{y y}^{\infty} \sqrt{\pi R}
$$

Irwin's formula in plane strains relates the stress intensity factor to the rate of energy release $G(\ell)$ :

$$
G(\ell)=-\frac{\partial U_{p}}{\partial \ell}=\frac{1-v^{2}}{Y} K_{I}^{2}
$$

$G_{i n c}(\ell)$ is readily obtained by integration:

$$
G_{i n c}(\ell)=\frac{1-v^{2}}{Y \ell} \int_{0}^{\ell} K_{I}^{2}(u) d u
$$

which yields, with $\lambda=\ell / R$ :

$$
G_{i n c}(\ell)=\Gamma\left(\frac{1}{21.29 \lambda}\left(1-\frac{1}{(1+\lambda)^{4.6}}\right)-\frac{1}{4.63(1+\lambda)^{5.6}}\right) \quad \text { with } \quad \Gamma=\frac{\pi R \sigma_{y y}^{\infty 2}\left(1-v^{2}\right)}{Y}
$$

Following (12), the length of the expected nucleated crack is $\ell^{*} \approx R / 3$. The critical loading level $\sigma_{y y}^{\infty}{ }^{*}$ that

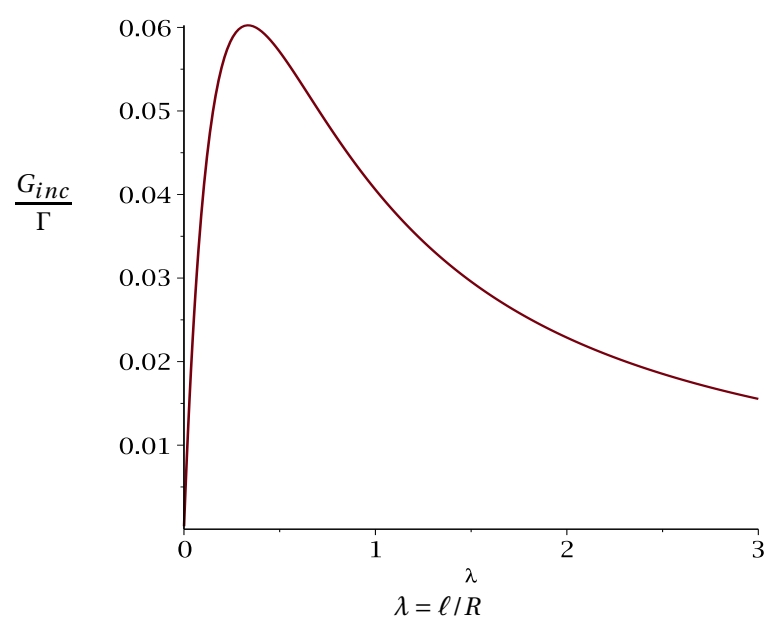

Fig. 10 Normalized rate of energy release $G_{i n c} / \Gamma$ associated with the nucleation of a tensile crack along $O y$ as a function of $\lambda=\ell / R$ for a uniaxial compressive remote stress $\sigma_{y y}^{\infty}$

induces nucleation is determined by the condition $G_{c} \approx 0.06 \Gamma$, that is:

$$
\sigma_{y y}^{\infty}=\frac{-1}{\sqrt{0.19\left(1-v^{2}\right)}} \sqrt{\frac{G_{c} Y}{R}}
$$

For further use, let us introduce the characteristic stress $\Sigma=-\sqrt{G_{c} Y / R}$. The critical compressive stress for mode I failure thus reads:

$$
\sigma_{y y}^{\infty *}=\frac{2.29 \Sigma}{\sqrt{1-v^{2}}}
$$

In the following, $\Sigma$ will be assumed to be a constant independant of $v$. 


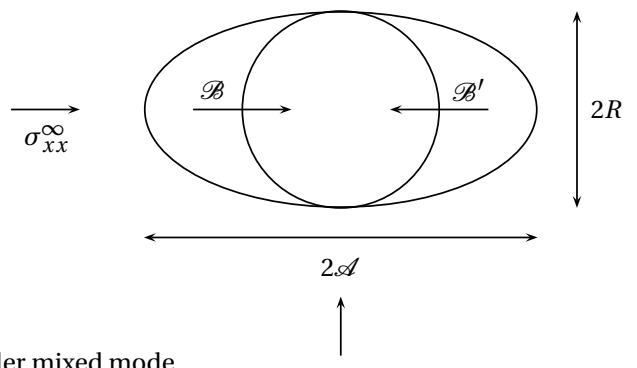

Fig. 11 failure mechanism under mixed mode

\subsection{A first attempt for modelling breakouts}

In the case of two compressive remote principal stresses $\sigma_{x x}^{\infty}, \sigma_{y y}^{\infty}<0$, the mechanism of section 4.2 is not promoted or even inhibited since tensile stresses along the $O y$ axis are weaker or even vanish (if $\sigma_{x x}^{\infty} / \sigma_{y y}^{\infty} \geq$ 1/3). As already stated, engineering practice mentions the occurence of breakouts. As an attempt to account for some failure patterns, a simple elliptical model is considered at figure 11. For simplicity, asymptotic displacements $\boldsymbol{\xi} \rightarrow \boldsymbol{\varepsilon}^{\infty} \cdot \boldsymbol{x}$ are prescribed at infinity $(|\boldsymbol{x}| \rightarrow \infty)$. We again restrict to the case of no stress acting on the cavity wall $(\eta=0)$. Accordingly, the potential energy is identical to the elastic energy and is obtained by integration of the volume density $\frac{1}{2} \varepsilon: \mathbb{C}: \varepsilon$. The basic assumption is the nucleation of an elliptic crack along the boundary of an ellipse with semi-axis $R$ in the direction of $O y$ and semi-axis $\mathscr{A}>R$ in the direction of $O x$. This means that the circular disc $\mathscr{D}$ and the ellipse $\mathscr{E}$ are tangent to each other. The mechanism consists of an inward displacement of the two blocks $\mathscr{B}$ and $\mathscr{B}^{\prime}$. This releases the stresses on the elliptic boundary of the surrounding medium and the stresses in blocks $\mathscr{B}$ and $\mathscr{B}^{\prime}$ as well as the corresponding elastic energy. The first task is to evaluate the elastic energy release involved in this mechanism. This is detailed for completedness at section 5.1 and yields

$$
G_{i n c}=\frac{1}{2 \ell} \varepsilon^{\infty}: \mathbb{C}:\left(|\mathscr{E}|\left(\mathbb{\square}-\mathbb{P}^{\mathscr{E}}: \mathbb{C}\right)^{-1}-|\mathscr{D}|\left(\llbracket-\mathbb{P}^{\mathscr{D}}: \mathbb{C}\right)^{-1}\right): \varepsilon^{\infty}
$$

in which $\ell$ denotes the perimeter of the ellipse $\mathscr{E} . \mathbb{P}^{\mathscr{E}}$ and $\mathbb{P}^{\mathscr{D}}$ are the Hill tensors of the ellipse $\mathscr{E}$ and of the disc $\mathscr{D}$. It can be shown that $G_{i n c}$ is almost a linear function of $\mathscr{A}$ and is equivalent of $\frac{\Gamma \mathscr{A}}{2^{1 / 3} \pi R}$ for $R / \mathscr{A} \ll 1$. Nevertheless, considering for instance a uniaxial stress state at infinity like in the previous section, it is readily seen from (42) that the normal stress along the ellipse has the sign of the remote stress $\sigma_{y y}^{\infty}$. This means that the expression given in (55) is only relevant in case of remote traction. In other words, the class of perturbations that are likely to take place in the context of remote compressive stress states must be based solely on mode II.

\subsection{Mode II cracks induced by an arbitrary remote stress}

In the following, the asymptotic boundary conditions are written on a circle of radius $L \gg R$ (see figure 12 (left)). Due to symmetry conditions it is possible to restrict the analysis to one quarter of space. This domain, denoted by $\Omega$, is defined by the hollow quarter of disk $B B^{\prime} C^{\prime} C$ (figure 12 (right)).

In the line of the method presented at section 2, we have to define the space of virtual displacements of the variational problem (13) and (14). To do so, we assume that the perturbation induced by nucleation is confined in a finite domain $\mathscr{T}_{\ell} \subset \Omega$, delimited by the points $B, P$ and $A(\ell=|A P|)$. In other words, it is assumed that the nucleation does not induce any change outside of $\mathscr{T}_{\ell}$.

Since the perturbation has a finite support in the vicinity of the wellbore, the nature of the asymptotic boundary conditions (given stresses or given displacements) is unimportant. Hereafter, the derivation of the potential energy is presented in the framework of given stresses $\boldsymbol{\sigma}^{\infty}$ at infinity.

Let $\xi^{0}, \boldsymbol{\varepsilon}^{0}$ and $\boldsymbol{\sigma}^{0}$ respectively denote the displacement, strain and stress tensors, solutions of the elastic 
problem before the crack nucleation (superscript 0 ). The elastic energy $W_{e}$ stored within $\Omega$ is defined as:

$$
W_{e}^{(0)}=\frac{1}{2} \int_{\Omega} \varepsilon^{0}: \mathbb{C}: \varepsilon^{0} d V
$$

and the virtual work of the prescribed forces in the solution displacement $\xi^{0}$ is defined as:

$$
\Phi^{(0)}=\int_{B^{\prime}}^{C^{\prime}} \boldsymbol{\sigma}^{\infty} \cdot \boldsymbol{e}_{r} \cdot \xi^{0} d S+\int_{C}^{B} \eta \boldsymbol{\sigma}^{\infty} \cdot\left(-\boldsymbol{e}_{r}\right) \cdot \xi^{0} d S
$$

After the crack nucleation, $\boldsymbol{\xi}^{0}, \boldsymbol{\varepsilon}^{0}$ and $\boldsymbol{\sigma}^{0}$ still define the elastic solution in $\Omega / \mathscr{T}_{\ell}$. The displacement, strain and stress tensors, solutions of the elastic problem in the perturbated domain $\mathscr{T}_{\ell}$ are:

$$
\boldsymbol{\xi}=\xi^{0}+\delta \boldsymbol{\xi} \quad ; \quad \boldsymbol{\varepsilon}=\boldsymbol{\varepsilon}^{0}+\delta \boldsymbol{\varepsilon} \quad ; \quad \boldsymbol{\sigma}=\boldsymbol{\sigma}^{0}+\delta \boldsymbol{\sigma}
$$

where $\delta \boldsymbol{\xi}, \delta \boldsymbol{\varepsilon}$ and $\delta \boldsymbol{\sigma}$ represent the perturbation fields due to the crack nucleation. The elastic energy stored within $\Omega$ after nucleation is defined as (superscript $\ell$ ):

$$
W_{e}^{(\ell)}=\frac{1}{2} \int_{\Omega / \mathscr{T}_{\ell}} \varepsilon^{0}: \mathbb{C}: \varepsilon^{0} d V+\frac{1}{2} \int_{\mathscr{T}_{\ell}} \boldsymbol{\varepsilon}: \mathbb{C}: \boldsymbol{\varepsilon} d V
$$

which may be rearranged in order to give:

$$
W_{e}^{(\ell)}=W_{e}^{(0)}+\frac{1}{2} \int_{\mathscr{T}_{\ell}} \delta \boldsymbol{\varepsilon}: \mathbb{C}: \delta \boldsymbol{\varepsilon} d V+\int_{\mathscr{T}_{\ell}} \boldsymbol{\varepsilon}^{0}: \mathbb{C}: \delta \boldsymbol{\varepsilon} d V
$$

Using the theorem of virtual work to the couple $\left(\boldsymbol{\sigma}^{0}=\mathbb{C}: \boldsymbol{\varepsilon}^{0}, \delta \boldsymbol{\varepsilon}\right)$, the last term in the right handside of (60) may be rewritten as:

$$
\int_{\mathscr{T}_{\ell}} \boldsymbol{\varepsilon}^{0}: \mathbb{C}: \delta \boldsymbol{\varepsilon} d V=\int_{A}^{B} \eta \boldsymbol{\sigma}^{\infty} \cdot\left(-\boldsymbol{e}_{r}\right) \cdot \delta \boldsymbol{\xi} d S+\int_{P}^{A} \boldsymbol{N} \cdot \boldsymbol{\sigma}^{0} \cdot \delta \boldsymbol{\xi} d S
$$

where $\boldsymbol{N}$ is the outward unit normal vector of domain $\mathscr{T}_{\ell}$ along the line segment $[P, A]$. Combining (60) and (61) eventually yields:

$$
W_{e}^{(\ell)}=W_{e}^{(0)}+\frac{1}{2} \int_{\mathscr{T}_{\ell}} \delta \boldsymbol{\varepsilon}: \mathbb{C}: \delta \boldsymbol{\varepsilon} d V+\int_{A}^{B} \eta \boldsymbol{\sigma}^{\infty} \cdot\left(-\boldsymbol{e}_{r}\right) \cdot \delta \boldsymbol{\xi} d S+\int_{P}^{A} \boldsymbol{N} \cdot \boldsymbol{\sigma}^{0} \cdot \delta \boldsymbol{\xi} d S
$$

In the cracked configuration, the virtual work of the prescribed forces reads:

$$
\Phi^{(\ell)}=\int_{B^{\prime}}^{C^{\prime}} \boldsymbol{\sigma}^{\infty} \cdot \boldsymbol{e}_{r} \cdot \xi^{0} d S+\int_{C}^{A} \eta \boldsymbol{\sigma}^{\infty} \cdot\left(-\boldsymbol{e}_{r}\right) \cdot \xi^{0} d S+\int_{A}^{B} \eta \boldsymbol{\sigma}^{\infty} \cdot\left(-\boldsymbol{e}_{r}\right) \cdot\left(\xi^{0}+\delta \boldsymbol{\xi}\right) d S
$$

Taking advantage of (57), the latter may also be written:

$$
\Phi^{(\ell)}=\Phi^{(0)}+\int_{A}^{B} \eta \boldsymbol{\sigma}^{\infty} \cdot\left(-\boldsymbol{e}_{r}\right) \cdot \delta \boldsymbol{\xi} d S
$$

The potential energy variation in the nucleation process is then derived from (62) and (64):

$$
U_{p}^{\ell}\left(\xi^{0}+\delta \boldsymbol{\xi}\right)-U_{p}^{0}\left(\xi^{0}\right)=\left(W_{e}^{(\ell)}-W_{e}^{(0)}\right)-\left(\Phi^{(\ell)}-\Phi^{(0)}\right)=\frac{1}{2} \int_{\mathscr{T}_{\ell}} \delta \boldsymbol{\varepsilon}: \mathbb{C}: \delta \boldsymbol{\varepsilon} d V+\int_{P}^{A} \boldsymbol{N} \cdot \boldsymbol{\sigma}^{0} \cdot \delta \boldsymbol{\xi} d S
$$

This expression can now be introduced into the variational problem (14). Note that the stress field in the uncracked configuration was presented at section 4.1. The perturbation displacement field $\delta \xi$ in the domain $\mathscr{T}_{\ell}$ has to be optimized. 

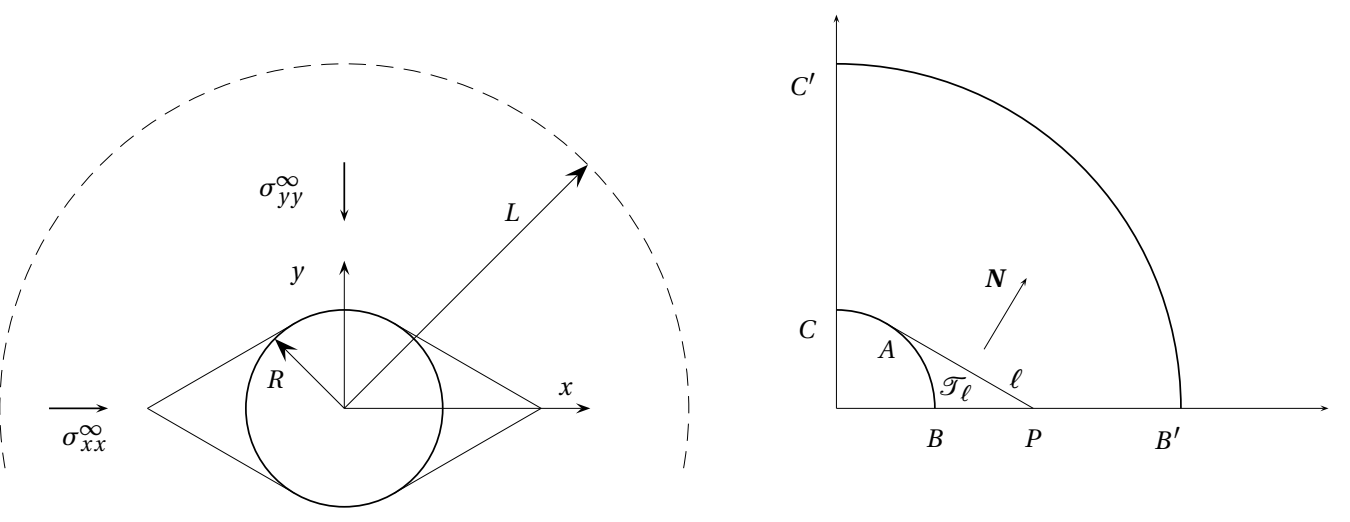

Fig. 12 failure mechanism in mode II

\subsubsection{The set of kinematically admissible perturbations}

As previously mentionned, the nucleation of a crack of length $\ell$ is associated with a perturbation of the displacement field within a domain located in the vicinity of the wellbore wall. For symmetry reasons, only one quarter of the perturbation area, namely $\mathscr{T}_{\ell}$, may be considered. The latter is bounded by the crack, namely the segment $P A$ with length $\ell=|\boldsymbol{A P}|$, the segment $B P(h=|\boldsymbol{B P}|)$ on the symmetry axis $O x$ and the circle arc $\overparen{B A}$ along the wellbore of radius $R$ (see figure 12). The segment $P A$ is tangent to the circle. Note that a wider class of failure mechanisms in which $P A$ is not necessarily tangent to the circle was also investigated and this revealed that the tangent case provides the optimum of this class. The geometry of $\mathscr{T}_{\ell}$ is therefore characterized by $\ell$ or by the angle $\beta=\widehat{A P B}$ satisfying to the condition $\tan \beta=R / \ell(\beta \in[0, \pi / 2[)$. Introducing the dimensionless parameter $\chi=\ell / R$, the surface area of $\mathscr{T}_{\ell}$ is a function of $\chi$ :

$$
S(\chi)=\frac{R^{2}}{2} \mathscr{S}(\chi) \quad ; \quad \mathscr{S}(\chi)=\chi-\arctan (\chi)
$$

The crack-induced perturbation is accounted for by the perturbation displacement field $\delta \xi$ within the domain $\mathscr{T}_{\ell}$. Its definition has to be compatible with the non-perturbated displacement field $\xi^{0}$ in $\Omega / \mathscr{T}_{\ell}$. Assuming a crack nucleation mechanism in mode $I I$, a discontinuity of $\delta \xi$ along the segment $P A$ is expected. This implies the following condition:

$$
\forall M \in P A \quad \delta \boldsymbol{\xi} \cdot \boldsymbol{N}=0
$$

Moreover, the symmetry condition along $(0 x)$ yields a second condition on $\delta \xi$ :

$$
\forall M \in P B \quad \delta \boldsymbol{\xi} \cdot \boldsymbol{e}_{y}=0
$$

For the sake of simplicity, we first consider an homogeneous transformation. The perturbation displacement field $\delta \xi$ is therefore defined by its gradient $\nabla(\delta \xi)$ as:

$$
\forall M \in \mathscr{T}_{\ell} \quad, \quad \delta \boldsymbol{\xi}=\nabla(\delta \boldsymbol{\xi}) \cdot \boldsymbol{P} \boldsymbol{M}
$$

In order to characterize this homogeneous transformation, the perturbation displacements $\delta \boldsymbol{\xi}_{A}\left(\right.$ resp. $\left.\delta \boldsymbol{\xi}_{B}\right)$ of point $A$ (resp. $B$ ) are introduced:

$$
\delta \boldsymbol{\xi}_{A}=\lambda_{A} \boldsymbol{P A} ; \delta \boldsymbol{\xi}_{B}=\lambda_{B} \boldsymbol{P B}
$$

where the longitudinal deformations $\lambda_{A}$ and $\lambda_{B}$ appear as two degrees of freedom likely to be optimized. In these conditions, it is readily seen that the gradient of the perturbation displacement field reads:

$$
\nabla(\delta \xi)=\sqrt{1+\chi^{2}}\left(\frac{\lambda_{A}}{\ell} \boldsymbol{P} \boldsymbol{A} \otimes \boldsymbol{e}_{y}+\lambda_{B} \boldsymbol{e}_{x} \otimes \boldsymbol{N}\right)
$$


Consequently, the perturbation strain field within the domain $\mathscr{T}_{\ell}$ is simply derived as:

$$
\delta \boldsymbol{\varepsilon}=\left(\begin{array}{cc}
\lambda_{B} & \frac{\chi}{2}\left(\lambda_{B}-\lambda_{A}\right) \\
\frac{\chi}{2}\left(\lambda_{B}-\lambda_{A}\right) & \lambda_{A}
\end{array}\right)_{\left(\boldsymbol{e}_{x}, \boldsymbol{e}_{y}\right)}
$$

\subsection{2 the associated incremental energy release rate}

Taking advantage of (42), (68), (70) and (71), the potential variation (65) involved in the crack nucleation process may be derived. The solution of the variational problem (14) amounts to the optimization of the parameters $\lambda_{A}$ and $\lambda_{B}$ which yields:

$$
\left\{\begin{array}{l}
\lambda_{A}=\frac{(1+v)\left((1-2 v) \chi^{2}+2(1-v)\right)}{Y\left(1+\chi^{2}\right)^{2} \mathscr{S}}\left(\lambda_{d} \sigma_{d}^{0}+\lambda_{m} \sigma_{m}^{0}\right) \\
\lambda_{B}=\frac{(1+v)\left((1-2 v) \chi^{2}-2 v\right)}{Y\left(1+\chi^{2}\right)^{2} \mathscr{S}}\left(\lambda_{d} \sigma_{d}^{0}+\lambda_{m} \sigma_{m}^{0}\right)
\end{array}\right.
$$

where the following dimensionless functions of $\chi$ and $\eta$ have been introduced:

$$
\lambda_{d}(\chi)=2 \mathscr{S}(1-\eta)\left(1-\chi^{2}\right)+(2-\eta) \chi^{3}-2(1-\eta) \chi \ln \left(1+\chi^{2}\right) \quad ; \quad \lambda_{m}(\chi)=(\eta-1)\left(1+\chi^{2}\right) \mathscr{S}
$$

The energy release reads:

$$
G_{i n c}(\chi)=\frac{R(1+v)\left((1-2 v) \chi^{2}+2(1-v)\right)}{2 Y \chi\left(1+\chi^{2}\right)^{3} \mathscr{S}}\left(\lambda_{d} \sigma_{d}^{0}+\lambda_{m} \sigma_{m}^{0}\right)^{2}
$$

In order to analyse the effect of the anisotropy of the remote loading, the ratio $\kappa=\sigma_{x x}^{\infty} / \sigma_{y y}^{\infty}(\kappa \in[0, \infty[)$ is introduced, the isotropic case being defined by $\kappa=1$. The incremental energy release rate then reads:

$$
G_{i n c}(\chi)=\frac{R(1+v)\left((1-2 v) \chi^{2}+2(1-v)\right)}{8 Y \chi\left(1+\chi^{2}\right)^{3} \mathscr{S}}\left(\lambda_{d}(\kappa-1)+\lambda_{m}(\kappa+1)\right)^{2}\left(\sigma_{y y}^{\infty}\right)^{2}
$$

For practical purpose, the normalized incremental energy release rate is also introduced as:

$$
\bar{G}_{i n c}(\chi)=\frac{G_{i n c}}{\left(R\left(\sigma_{y y}^{\infty}\right)^{2} / Y\right)}=\frac{(1+v)\left((1-2 v) \chi^{2}+2(1-v)\right)}{8 \chi\left(1+\chi^{2}\right)^{3} \mathscr{S}}\left(\lambda_{d}(\kappa-1)+\lambda_{m}(\kappa+1)\right)^{2}
$$

The latter is represented as a function of $\chi$ for an anisotropic compressive remote stress before (resp. after) drilling on figure 13 (resp. figure 14).

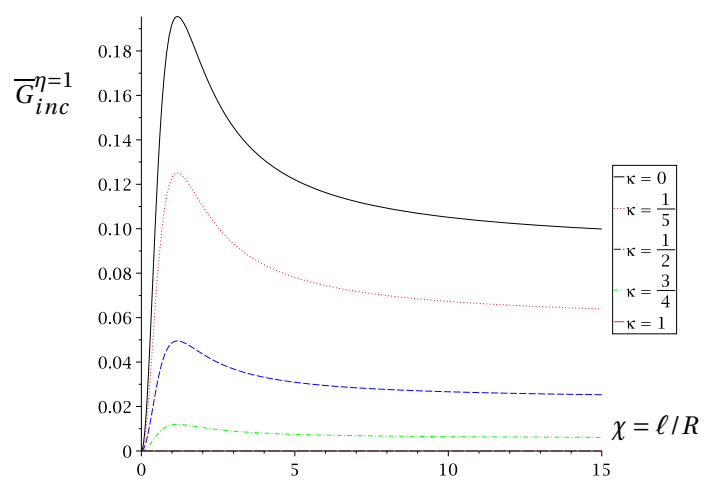

(a) $v=0.2$

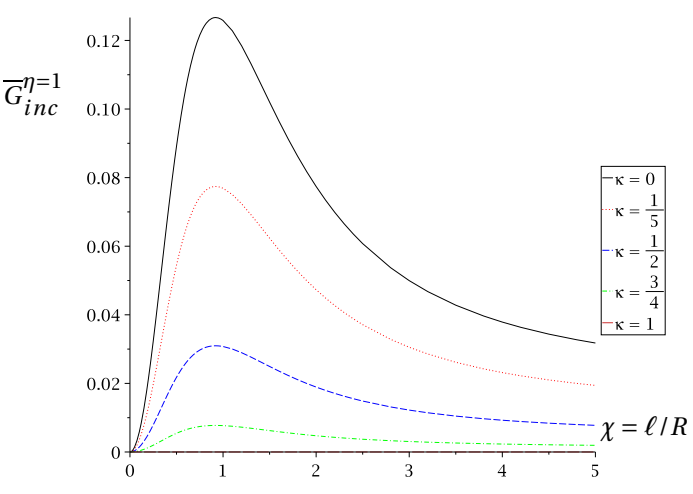

(b) $v=0.45$

Fig. $13 \bar{G}_{i n c}$ as a function of $\chi$ for an anisotropic compressive remote stress $(\kappa \in[0,1])$ before drilling $(\eta=1)$ 


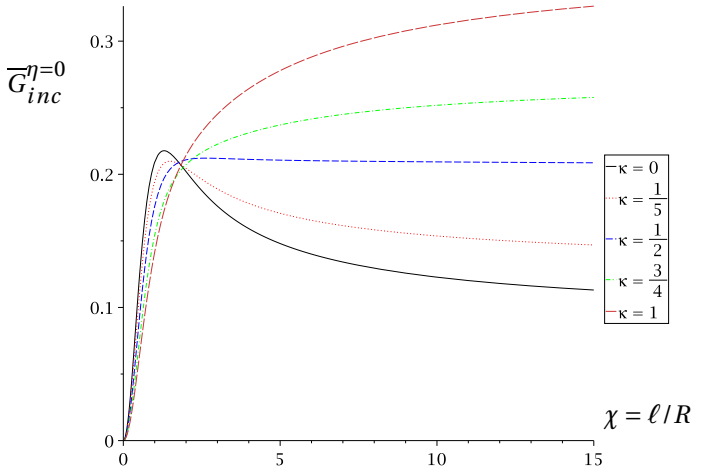

(a) $v=0.2$

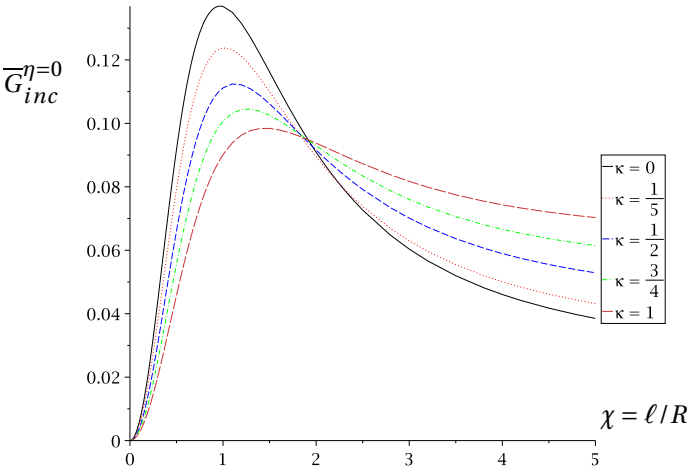

(b) $v=0.45$

Fig. $14 \bar{G}_{i n c}$ as a function of $\chi$ for an anisotropic compressive remote stress for $\eta=0(\kappa \in[0,1])$

\subsubsection{Comparison of failure modes in uniaxial compression}

The general framework proposed herein allows to compare the failure modes that have been discussed above. To illustrate this, we restrict to the situation of uniaxial compression along $O y(\kappa=0)$ at the end of drilling $(\eta=0)$. The critical stress in mode II is obtained according to (12) from (76):

$$
\max _{\chi} \bar{G}_{i n c}(\chi)=\left(\frac{\Sigma}{\sigma_{y y}^{\infty}}\right)^{2} \Leftrightarrow \sigma_{y y}^{\infty}=\frac{\Sigma}{\sqrt{\max _{\chi} \bar{G}_{i n c}(\chi)}}
$$

with $\Sigma=-\sqrt{G_{C} Y / R}$. This estimate is plotted together with the critical stress in mode I from (54) at figure 15 Interestingly, if the same value of the critical energy $G_{c}$ is used for both mode I and mode II, the latter

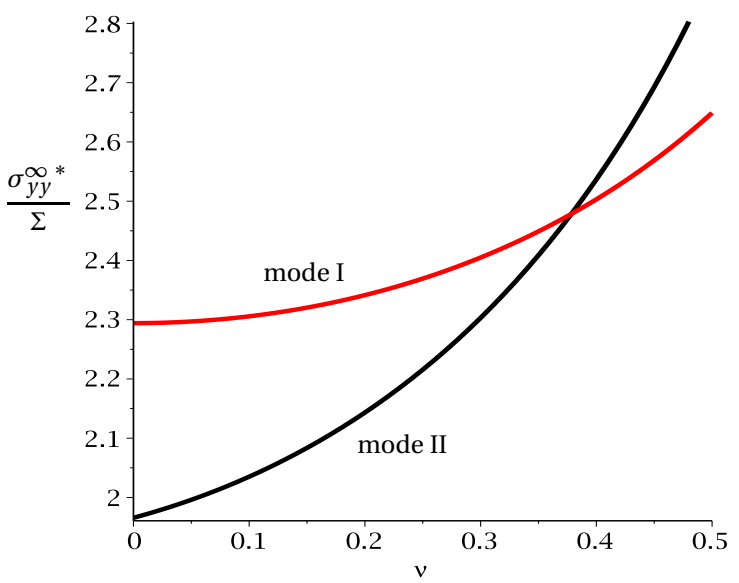

Fig. 15 Critical stress in uniaxial compression as a function of the Poisson ratio $v$ at the end of drilling $(\eta=0)$ : comparison between mode I and mode II

should be promoted for values of the Poisson ratio up to 0.37 . In other words, a breakout should be observed instead of a tensile crack.

\subsubsection{Geometry of the breakout}

The geometry of the breakout is characterized by the critical length $\ell^{*}$ or the corresponding ratio $\chi^{*}=\ell^{*} / R$. In the initial state before drilling $(\eta=1)$, the energy release $G_{\text {inc }}$ takes the form:

$$
\bar{G}_{i n c}^{\eta=1}(\chi)=\frac{(1+v)\left((1-2 v) \chi^{2}+2(1-v)\right) \chi^{5} \mathscr{S}}{2\left(1+\chi^{2}\right)}(1-\kappa)^{2}
$$




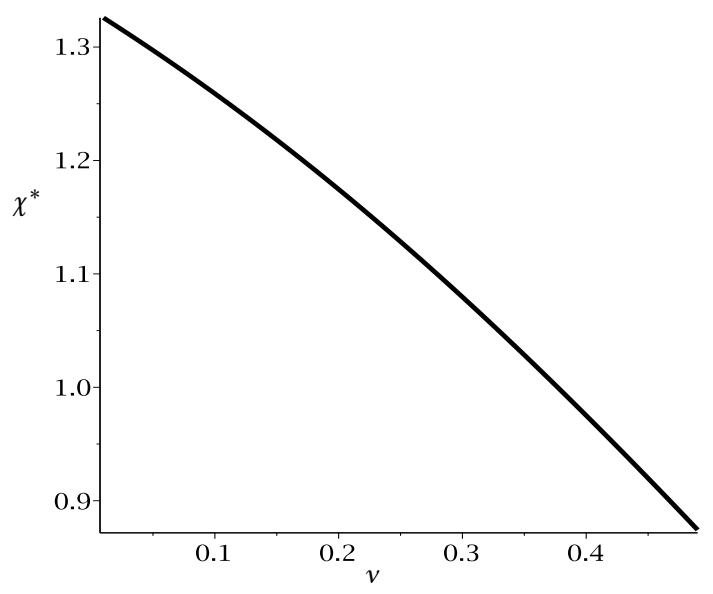

Fig. $16 \chi^{*}$ as a function of the Poisson ratio $v$ in the initial state $(\eta=1)$

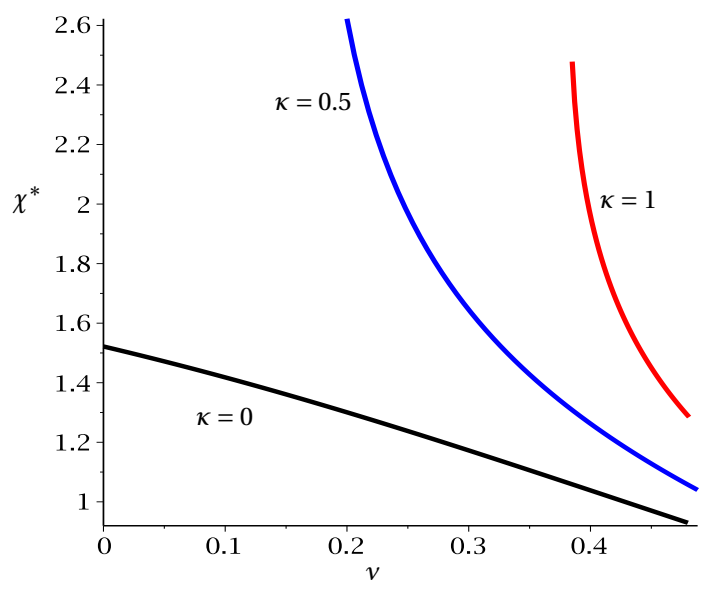

Fig. $17 \chi^{*}$ as a function of the Poisson ratio $v$ at the end of drilling $(\eta=0)$

The location of the maximum is clearly independant of the parameter $\kappa$ which measures the anisotropy of the remote stress state (see figure 13). It is plotted as a function of the Poisson ratio at figure 16.

In contrast, at the end of drilling $(\eta=0)$, the geometry of the breakout strongly depends on both the Poisson ratio and the stress anisotropy parameter $\kappa$ (see figure 17). For $\kappa \leq 0.5$, the optimal breakout has finite dimensions, that is, the nucleation of a crack with finite length is obtained. However, for $\kappa>0.5$, the curve $\chi^{*}(v)$ possesses a vertical asymptote for some value of the Poisson ratio that increases with $\kappa$. This means that a stable crack is only obtained for higher values of the Poisson ratio. Otherwise, for lower values of the Poisson ratio, the nucleation is not stable.

The same conclusions can be drawn from the plot of the function $G_{i n c}(\chi)$. The stability of the nucleation is associated with the existence of a maximum of the function $G_{i n c}(\chi)$. As can be seen on figure 14, the latter is always granted for $v=0.45$, irrespective of the anisotropy of the remote stress. In contrast, for $v=0.2$, there is a transition around $\kappa=1 / 2$ between a regime with or without maximum. Stable nucleation is therefore promoted by the anisotropy of the remote stress and by high values of the Poisson ratio.

\subsubsection{Critical stress of the breakout}

The discussion concerning the critical stress is controlled by the parameter $\eta$ which controls the level of drilling. Before drilling $(\eta=1)$, the expression (78) of $G_{i n c}$ together with (77) reveals that the critical stress is 
an increasing function of $\kappa$. It is related to the critical stress in uniaxial compression $(\kappa=0)$ by:

$$
\sigma_{y y}^{\infty *}=\frac{\sigma_{y y}^{\infty}{ }_{\mid \kappa=0}}{1-\kappa}
$$

In particular, the infinite value obtained at the limit $\kappa \rightarrow 1$ indicates, as expected, that no failure can take place before drilling in the case of an isotropic remote stress state. Besides, at some given value of $\kappa$, the critical stress is an increasing function of the Poisson ratio (see figure 18). At the end of drilling $(\eta=0)$,

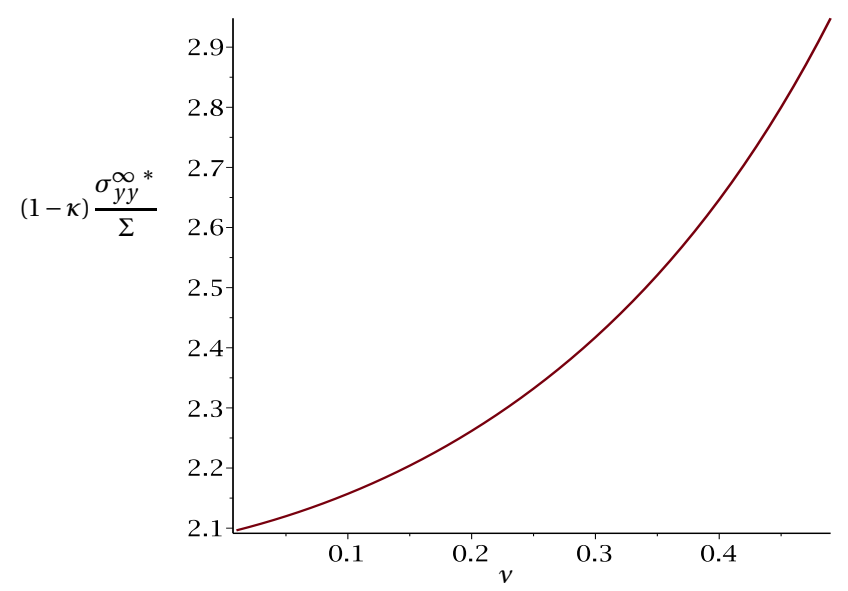

Fig. 18 Critical stress $\sigma_{y y}^{\infty}{ }_{\mid \kappa=0}$ as a function of the Poisson ratio $v$ before drilling $(\eta=1)$

the critical stress is still an increasing function of the Poisson ratio but the influence of the anisotropy of the remote stress state is less noticeable. The critical stress is plotted as a function of the Poisson ratio for $\kappa=0,0.25$ and 0.5 at figure 19. For values of $\kappa>0.5$, a stable nucleation is observed only for high values of the

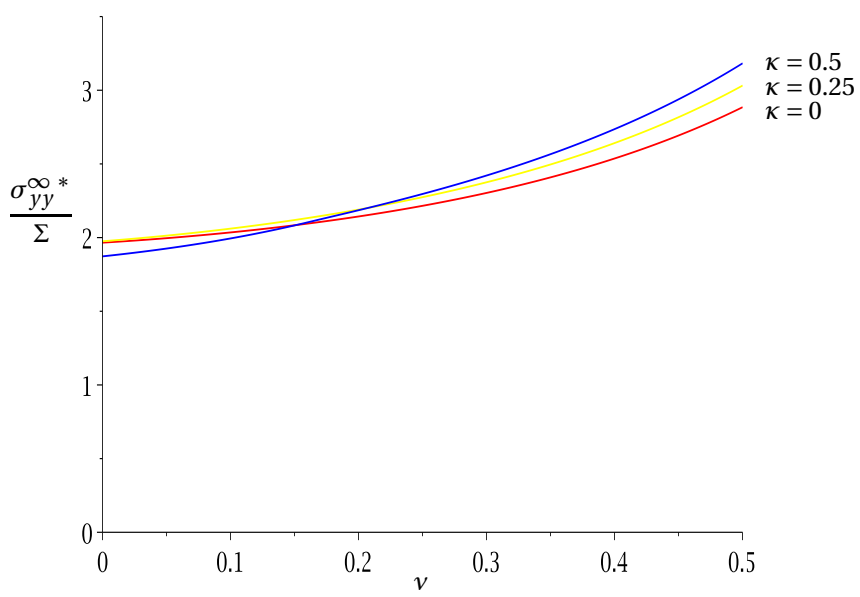

Fig. 19 Critical stress $\sigma_{y y}^{\infty}{ }^{*}$ as a function of the Poisson ratio $v$ at the end of drilling $(\eta=0)$

Poisson ratio. The critical stress is derived from the maximum of $G_{i n c}$ or from the asymptote at $\chi \rightarrow \infty$ (see figure 20). The general property of critical stress increase with $v$ is clearly confirmed. The effect of drilling on the stability of the wellbore is clearly an issue as regards engineering practice. It is herein modelled by the decrease from 1 to 0 of the parameter $\eta$. The influence of the latter is strongly dependent on the anisotropy of the remote stress state. For an isotropic one $(\kappa=1)$, drilling has a very strong influence. In this case, the 


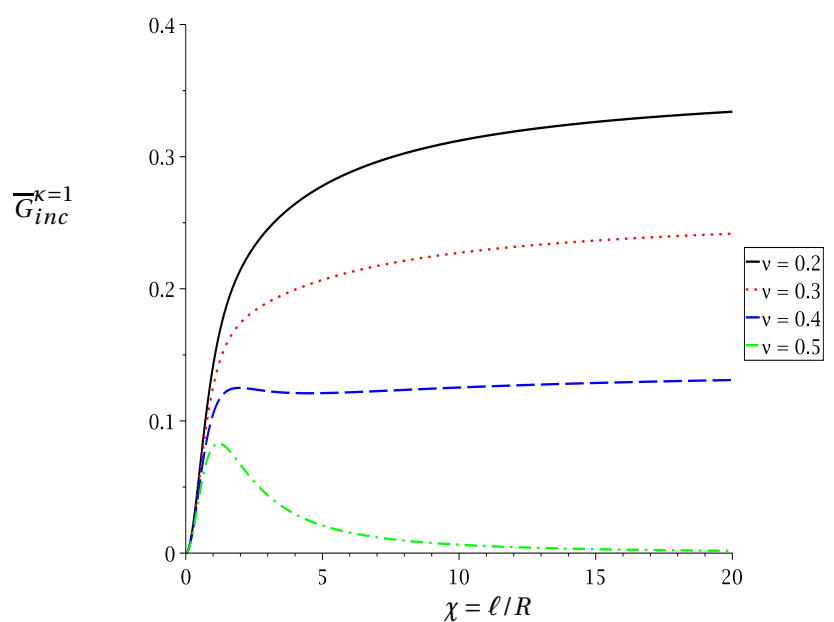

Fig. 20 Normalized $\bar{G}_{i n c}$ as a function of $\chi$ and $v$ for an isotropic $(\kappa=1)$ compressive remote stress $(\eta=0)$. For $v=0.4,0.5$, the half-angle at the apex of the cone is $\beta^{*}=\arctan \left(1 / \chi^{*}\right)$.

general expression (76) simplifies:

$$
\bar{G}_{i n c}^{\kappa=1}(\chi)=\frac{(1+v)\left((1-2 v) \chi^{2}+2(1-v)\right) \mathscr{S}}{2 \chi\left(1+\chi^{2}\right)}(1-\eta)^{2}
$$

Recalling (77), this means that the critical stress reads:

$$
\sigma_{y y}^{\infty *}=\frac{\sigma_{y y}^{\infty *}{ }_{\mid \eta=0}^{\infty}}{1-\eta}
$$

which provides a simple analytical model for the asymptote observed at figure 21 in the case $\kappa=1$. In contrast, the effect of drilling is almost negligible in the case of uniaxial compression $(\kappa=0)$.

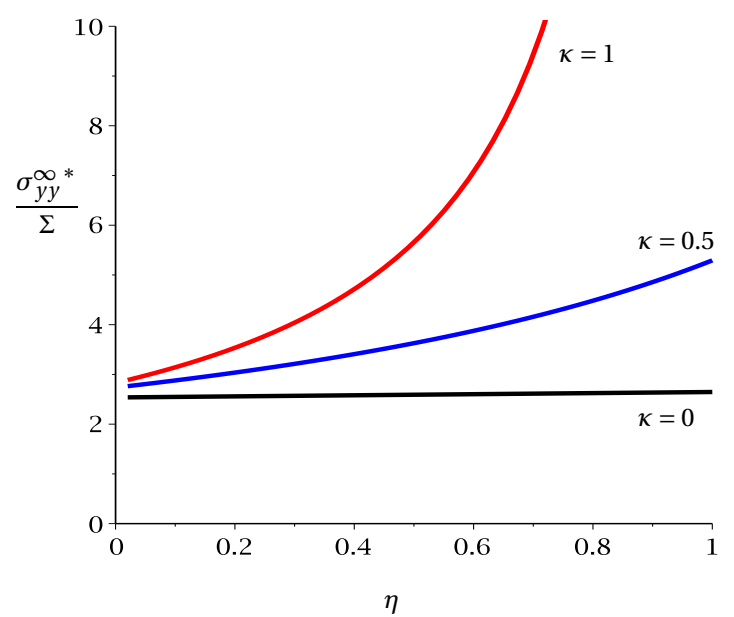

Fig. 21 Critical stress $\sigma_{y y}^{\infty}{ }^{*}$ as a function of $\eta$ for different anisotropy levels $(v=0.4)$

\subsubsection{Comparison with finite element simulations}

As stated before, the results presented so far have been obtained from the solution to a variational problem defined by (14). The space of kinematically admissible perturbations was described at section 4.4.1. It depends on a geometrical parameter, namely the location of point $P$ on the $O x$ axis (see figure 12). The simplifying assumption was that the k.a. perturbations were defined by a homogeneous transformation within 
the perturbated zone $\mathscr{T}_{\ell}$. Owing to the symmetry conditions, each k.a. perturbation is therefore characterized by the two longitudinal deformations $\lambda_{A}$ and $\lambda_{B}$ introduced in (69). As stated at the end of section 2, if the space of k.a. perturbations is enriched, a more accurate (smaller) estimate of the critical loading level is expected. In the present section, the idea is to keep the same geometry of the perturbated zone but to drop the assumption of homogeneous perturbation gradient. Instead, a finite element mesh of $\mathscr{T}_{\ell}$ is used to define the space of k.a. perturbations. The numerical results of the finite element simulations are compared to the results already presented at figure 21 . It confirms that the simplified model provides less accurate estimates of the critical stress. However, the discrepancy is weak enough and justifies the use of the analytical results derived at section 4.4.2.

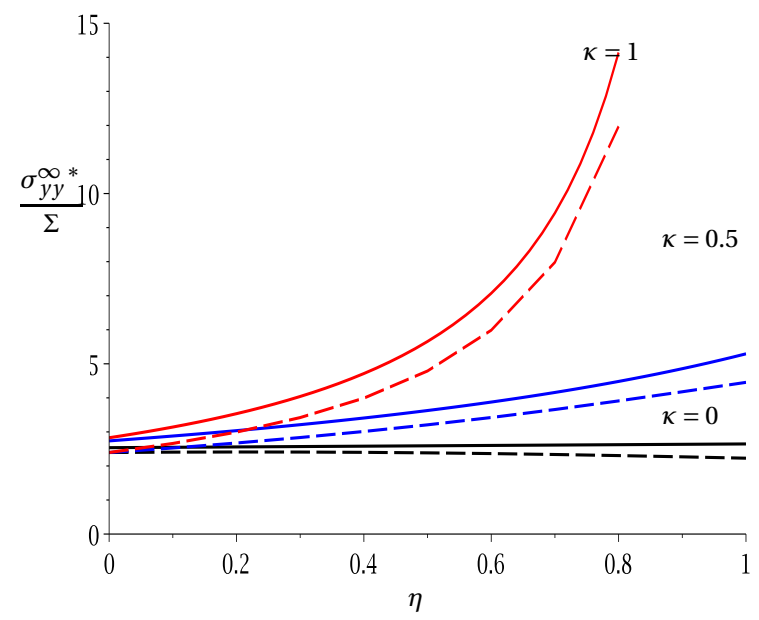

Fig. 22 Critical stress $\sigma_{y y}^{\infty}{ }^{*}$ as a function of $\eta$ for different anisotropy levels $(v=0.4)$. The dashed lines present the results of the finite element simulations.

\subsection{Brittle failure versus limit analysis}

The failure mechanisms considered in this paper are based on the material brittleness. In contrast, engineering practice often deals with wellbore stability in the context of perfect elastoplastic behavior [30]. If this latter model should be preferred, the appropriate framework is the theory of limit analysis (e.g. [29]). In this section, we briefly address the issue of the wellbore stability with this point of view and we emphasize the differences between the conclusions that can be drawn when using the physical assumptions of brittle or ductile failures.

To begin with, let us consider the system before drilling $(\eta=1)$. The critical stress predicted by the brittle failure approach is given in (79). As far as limit analysis is concerned, the conclusion is quite the opposite (except for the isotropic case $\kappa=1$ ). Indeed, let us consider an application of the static theorem. Clearly enough, a uniform stress field equal to the same stress state that is applied at infinity and in the wellbore is statically admissible. Irrespective of the strength criterion, if the remote stress state is plastically admissible, the considered (uniform) stress field is both plastically and statically admissible. In other words, by application of the static theorem of limit analysis, if a ductile behavior is assumed, no failure can take place under application of a plastically admissible remote stress state.

Let us now consider the system after drilling $(\eta=0)$. It is recalled that the cavity wall is free of stress which can be regarded as the most unfavorable situation as far as stability is concerned. A glance at figure 21 recalls that the brittle failure approach always predicts some failure threshold, irrespective of the anisotropy ratio $k$ of the remote stress state. In particular, for an isotropic remote stress state, the failure threshold can be derived from the maxima (resp. asymptotes) of figure 20, depending on the Poisson ratio. Let us now consider the same question with the point of view of limit analysis. For simplicity, we shall restrict to the 
isotropic case $\kappa=1$ for which a very simple analytic solution can be derived in the case of a Tresca strength criterion

$$
\max _{i, j}\left|\sigma_{i}-\sigma_{j}\right|-\sigma_{0} \leq 0
$$

where $\sigma_{0}$ is a material constant and $\sigma_{i}$ are the principal stresses. For a hollow cylinder with inner radius $R$ and outer radius $R^{\prime}$ with a pressure $p$ applied on the outer boundary, the limit pressure reads [29]:

$$
p_{\text {lim }}=\sigma_{0} \log \frac{R^{\prime}}{R}
$$

Clearly enough, this means that the limit pressure is infinite in the case of an infinite medium. Once again, a rigorous application of limit analysis, which is the appropriate tool for dealing with ductile failure, yields a very different conclusion as compared to the brittle failure approach, since limit analysis concludes that $\kappa=1$ corresponds to a stable state. For these reasons, it is believed that the ductile model is questionable as regards the interpretation of breakouts.

\section{Conclusion}

In this paper, the nucleation of a crack in a saturated porous material is modelled as an adiabatic and undrained event. The thermodynamical analysis requires to attribute an internal energy to the region of space in which the crack is created. This amounts to considering the crack as a material system in which a full damage process takes place. With this point of view, it has been shown that a model based on isotropic damage sometimes fails to predict accurately the formation of a crack. This is in particular the case for tensile cracks starting from the edge of a circular hole initiated by a uniaxial compressive remote stress.

The thermodynamical analysis provides an energy criterion for nucleation that can be viewed as an alternative to the double criterion proposed by [14]. The geometrical parameters (orientation, length) of the crack and the level of the loading which triggers its nucleation are determined from the solution of a variational problem which consists in seeking the maximum of the incremental rate of potential energy release.

The loading level required for the nucleation of a fracture in mode I in a saturated porous material can be increased by the presence of the pore fluid if the latter exhibits a low compressibility. Physically, this is due to the fact that, in undrained conditions, the stiffness of the solid-fluid mixture incorporates a contribution of the fluid that vanishes only when the pore fluid pressure reaches the saturation vapour pressure.

In the case of a compressive remote stress state, the breakouts observed in the wellbores can be initiated by fracture nucleations in mode II. Within the assumption of an isotropic elastic behavior, the presence of a pore fluid does not affect such a failure mechanism. In contrast, limit analysis fails to predict an instability of wellbore under the same loading conditions.

In this paper, the breakout is delimited by two rectilinear cracks tangent to the wellbore and by a circular arc located on the boundary of the wellbore. The support of the perturbation induced by the crack nucleation is limited to this bounded domain. In the latter, the gradient of the transformation associated is homogeneous. The variational problem consists in the optimization of this gradient (2 kinematical parameters) and of the orientation of the cracks (1 parameter).

This simple analytical model compares favourably with a more sophisticated finite element optimization. It allows to perform a parametric analysis. In particular, it reveals that stable nucleation is promoted by the anisotropy of the remote stress and by high values of the Poisson ratio. 


\section{appendix}

\subsection{Energy release in the mechanism of figure 11}

Since the assumption is that the elastic energy in blocks $\mathscr{B}$ and $\mathscr{B}^{\prime}$ is completely released, it amounts to determine the difference of elastic energy between the two situations depicted at figure 23:
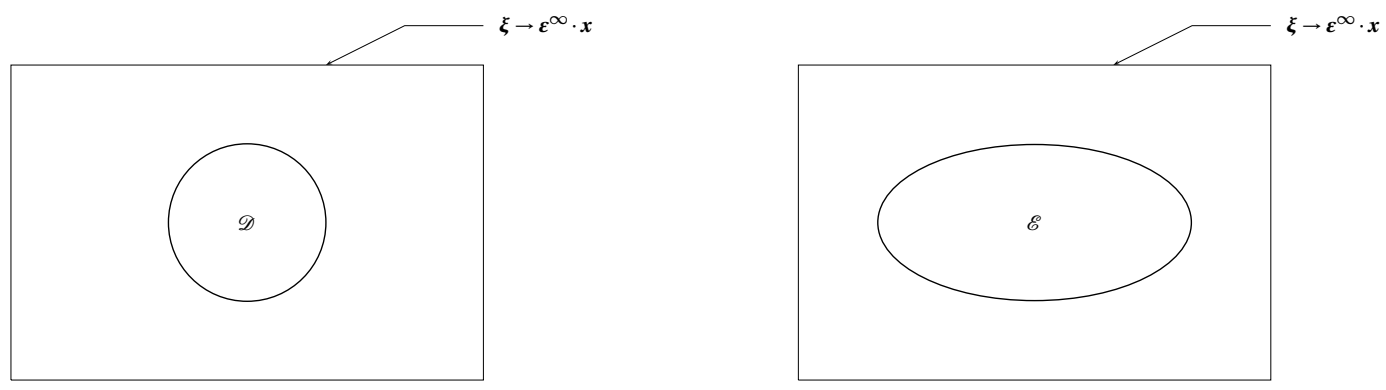

Fig. 23 Circular and elliptical cavities in infinite elastic medium with remote strain state

Prior to the nucleation, the strain field in the domain surrounding the circular hole is wellknown. After nucleation, the cavity has become the ellipse $\mathscr{E}$. The strain field in the domain surrounding the elliptical hole as well as in the elliptical cavity is the solution to the Dederichs-Zeller equation [26]:

$$
\boldsymbol{\varepsilon}+\Gamma^{\infty} *(\delta \mathbb{C}: \boldsymbol{\varepsilon})=\boldsymbol{\varepsilon}^{\infty}
$$

in which $\Gamma^{\infty}$ is the Green operator of the infinite medium (see e.g. [3]) associated with the elastic stiffness tensor $\mathbb{C} . \delta \mathbb{C}$ is equal to 0 outside of the ellipse $\mathscr{E}$ and is equal to $-\mathbb{C}$ in the ellipse. This implies that the strain state $\boldsymbol{\varepsilon}^{\mathscr{E}}$ in the elliptical cavity is uniform:

$$
\boldsymbol{\varepsilon}^{\mathscr{E}}=\left(\llbracket-\mathbb{P}^{\mathscr{E}}: \mathbb{C}\right)^{-1}: \boldsymbol{\varepsilon}^{\infty}
$$

in which $\mathbb{P}^{\mathscr{E}}$ is the Hill tensor of the ellipse. For any location $\boldsymbol{x}$ outside of the ellipse, it follows from (84) and (85) that

$$
\boldsymbol{\varepsilon}(\boldsymbol{x})=\boldsymbol{\varepsilon}^{\infty}+\left(\int_{\mathscr{E}} \Gamma^{\infty}\left(\boldsymbol{x}-\boldsymbol{x}^{\prime}\right) d S_{x^{\prime}}\right): \mathbb{C}: \boldsymbol{\varepsilon}^{\mathscr{E}}
$$

Clearly enough, the elastic strain field in the medium surrounding the ellipse is identical to the strain field that is used in the dilute scheme for the derivation of the so-called dilute estimate of the elastic stiffness. Note that the above equation (86) also holds for the particular case of the circular hole $\mathscr{D}$. Let us recall the expression of the dilute estimate $\mathbb{C}^{d i l}$ of the elastic stiffness of a porous medium in which the solid matrix has the elastic stiffness $\mathbb{C}$ and an elliptical pore space with volume fraction $f$ :

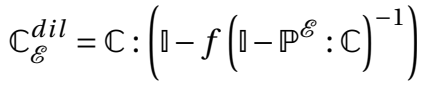

Our purpose is to determine the energy $\delta U_{p}^{\mathscr{E}}$ of the perturbation associated with an elliptical hole in an infinite uniform medium with respect to the uniform case. Let $\Omega_{L}$ denote the disc of radius $L$. What we look for is the limit

$$
\delta U_{p}^{\mathscr{E}}=\lim _{L \rightarrow \infty} \frac{1}{2} \varepsilon^{\infty}:\left(\mathbb{C}_{\mathscr{E}}^{d i l}-\mathbb{C}\right): \varepsilon^{\infty}\left|\Omega_{L}\right|
$$

It is readily derived from (87), with $f=|\mathscr{E}| /\left|\Omega_{L}\right|$ :

$$
\delta U_{p}^{\mathscr{E}}=-\frac{1}{2}|\mathscr{E}| \boldsymbol{\varepsilon}^{\infty}: \mathbb{C}:\left(\llbracket-\mathbb{P}^{\mathscr{E}}: \mathbb{C}\right)^{-1}: \boldsymbol{\varepsilon}^{\infty}
$$


Let us now return to the initial purpose namely the derivation of the energy release associated with the nucleation of the elliptic crack. Since it corresponds to the difference of potential energy between the circular and the elliptic case, it is readily derived from (88):

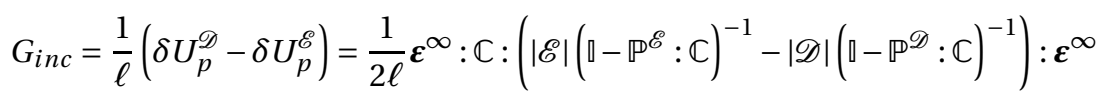

in which $\ell$ denotes the perimeter of the ellipse $\mathscr{E}$.

\subsection{The generalized Eshelby problem}

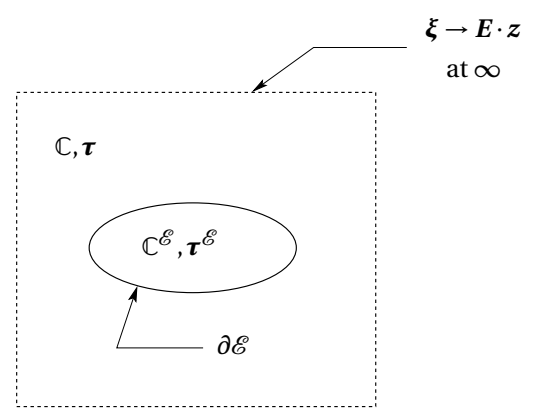

Fig. 24 The generalized Eshelby problem

Consider an infinite homogeneous linear elastic medium with stiffness tensor $\mathbb{C}$ in which the constitutive equation reads:

$$
\boldsymbol{\sigma}=\mathbb{C}: \boldsymbol{\epsilon}+\boldsymbol{\tau}
$$

The tensor $\boldsymbol{\tau}$ represents some uniform initial stress in the reference state. An ellipsoidal (resp. elliptic in plane strain conditions) domain $\mathscr{E}$ is embedded in this medium. The constitutive equation in $\mathscr{E}$ reads (uniform initial stress $\boldsymbol{\tau}^{\mathscr{E}}$ ):

$$
\boldsymbol{\sigma}=\mathbb{C}^{\mathscr{E}}: \boldsymbol{\epsilon}+\boldsymbol{\tau}^{\mathscr{E}}
$$

Under application of an asymptotic uniform strain $\boldsymbol{E}$ at infinity (figure 24), the strain induced in the domain $\mathscr{E}$ is uniform and reads (e.g. [3]):

$$
\boldsymbol{\epsilon}^{\mathscr{E}}=\left(\llbracket+\mathbb{P}:\left(\mathbb{C}^{\mathscr{E}}-\mathbb{C}\right)\right)^{-1}:\left(\boldsymbol{E}-\mathbb{P}:\left(\boldsymbol{\tau}^{\mathscr{E}}-\boldsymbol{\tau}\right)\right)
$$

where $\mathbb{P}$ is the Hill's tensor of $\mathscr{E}$ embedded in the homogenous medium with stiffness tensor $\mathbb{C}$.

\section{References}

1. Griffith A. A., The phenomenon of rupture and flow in solids, Philosophical Transactions of the Royal Society of London, vol. 221, $163-198,1921$.

2. Sneddon I. N. and Lowengrub M., Crack problems in the classical theory of elasticity, Wiley, 1969.

3. Dormieux, L. and Kondo, D., Micromechanics of fracture and damage, ISTE-Wiley, 2016.

4. Dormieux, L., Kondo, D. and Ulm F.-J. , Microporomechanics, Wiley, 2006.

5. Pham, K and Marigo, J.-J., Gradient damage models and their use to approximate brittle fracture, International Journal of Damage Mechanics, vol. 20, 4, 618-652, 2011.

6. Pham, K and Marigo, J.-J., From the onset of damage to rupture: construction of responses with damage localization for a general class of gradient damage models, Continuum Mechanics and Thermodynamics, vol. 25, 2, 147-171, 2013.

7. Marigo, J.-J.,Constitutive relations in Plasticity, Damage and Fracture Mechanics based on a work property, Nuclear Eng. Design, 114, 249-272, 1989.

8. Rice J.R., Thermodynamics of the quasi-static growth of Griffith cracks, Journal of the Mechanics and Physics of Solids, vol. 26, $61-78,1978$.

9. Sih G. S., Handbook of stress intensity factors, Lehigh University, Bethlehem, 1973

10. Kachanov M., Elastic solids with many cracks related problems, Academic Press, San Diego, 1993.

11. Budiansky B. and O'Connell R., Elastic moduli of a cracked solid, International Journal of Solids and Structures, vol.12, 81-97, 1976. 
12. Horii H. and Nemat-Nasser S., Overall elastic modulii with microcracks: load-induced anisotropy, Journal of the Mechanics and Physics of Solids, vol. 31, 155-171, 1983

13. Ponte-Castaneda P. and Willis J.R., The effect of spatial distribution on the effective behavior of composite materials and cracked media, Journal of the Mechanics and Physics of Solids, vol. 43, 12, 1919-1951, 1995.

14. Leguillon D., Strength or toughness: a criterion for crack onset at a notch. European Journal of Mechanics - A/Solids, vol. 21, 61-72, 2002.

15. Carlioz T., Nucléation et propagation de fissures en conditions anisotropes, Thèse de doctorat, Université Paris-Est, 2017.

16. Carlioz T., Dormieux L. and Lemarchand E., Thermodynamics of crack nucleation, Continuum Mechanics and Thermodynamics, 2020.

17. Coussy O., Mechanics and Physics of Porous Solids. Wiley, 2010.

18. Hill R., A general theory of uniqueness and stability in elastic-plastic solids, Journal of the Mechanics and Physics of Solids, vol. 6, 236-249, 1958

19. Adda-bedia, 2013. Stability and roughness of tensile cracks in disordered media, Physical Review E 88 (052402)

20. De Buhan P., 2007. Plasticité et calcul à la rupture, Eyrolles

21. Dormieux L., Lemarchand E., Kondo D., 2017. Mécanique des milieux continus, Ellipse

22. Dormieux L., Lemarchand E., CarliozT., Jeannin L., 2019 Crack nucleation in saturated porous media, International Journal for numerical and analytical methods in geomechanics

23. Jeannin L., Dormieux L., Carlioz T., 2019. Nucleation of thermal cracks at the wall of a rock mass, Mechanics Research Communications, to be published.

24. Leblond J.B., 2003. Mécanique de la rupture fragile et ductile, Hermès

25. Leguillon D., 2001. Strengh or toughness? A criterion for crack onset at a notch, Eur. J. Mech. A, Solids 21:61-72

26. Dederichs P.H. and Zeller R., Variational treatment of the elastic constants of disordered materials, Zeitscrift fur Physik, vol 259, 103-116, 1973

27. G. C. Sih, 1973. Handbook of Stress Intensity Factors for Researchers and Engineers, Lehigh Univ

28. Sammis C.G., Ashby M.F., 1986. The failure of brittle porous solids under compressive stress states, 34(3):511-526, 1986

29. Salençon J., 2013. Yield Design, Wiley 2013

30. Zoback M.D., 2007. Reservoir geomechanics, Cambridge University Press

31. J.C. Jaeger, N.G.W. Cook, 1969. Fundamentals of Rock Mechanics Methuen \& Co 